\title{
A framework for global reliability sensitivity analysis in the presence of multi-uncertainty
}

\author{
Max Ehre, Iason Papaioannou, Daniel Straub
}

Engineering Risk Analysis Group, Technische Universität München, 80333 München, Germany

\begin{abstract}
In reliability analysis with numerical models, one is often interested in the sensitivity of the probability of failure estimate to changes in the model input. In the context of multi-uncertainty, one whishes to separate the effect of different types of uncertainties. A common distinction is between aleatory (irreducible) and epistemic (reducible) uncertainty, but more generally one can consider any classification of the uncertain model inputs in two subgroups, type A and type B. We propose a new sensitivity measure for the probability of failure conditional on type B inputs. On this basis, we outline a framework for multi-uncertainty-driven reliability sensitivity analysis. A bi-level surrogate modelling strategy is designed to efficiently compute the new conditional reliability sensitivity measures. In the first level, a surrogate is constructed for the model response to circumvent possibly expensive evaluations of the numerical model. By solving a sequence of reliability problems conditional on samples of type B random variables, we construct a level 2-surrogate for the logarithm of the conditional probability of failure, using polynomial bases which allow to directly evaluate the variance-based sensitivities. The new sensitivity measure and its computation are demonstrated through two engineering examples.
\end{abstract}

Keywords: Reliability-Oriented Sensitivity Analysis, Multi-Uncertainty, Surrogate Modelling, Rare Event Simulation, Decision Support

\section{Introduction}

Reliability analysis is concerned with the evaluation of the probability of failure of an engineering system. The system can be described probabilistically in terms of the input random vector $\Theta$ with joint cumulative distribution function $(\mathrm{CDF}) F_{\boldsymbol{\Theta}}$ and a deterministic model $\mathcal{Y}$ mapping each $\boldsymbol{\Theta}$ to an output $Y=\mathcal{Y}(\boldsymbol{\Theta})$. The performance of the system can be assessed in terms of its limit-state function $g$. The limit-state function defines the failure modes of a system and by convention takes values below 0 in the failure domain, which is a subset of the entire input variable space $\Omega_{\Theta}$. The system probability of failure is given by [1]

$$
\mathbb{P}(F)=\mathbb{E}_{\Theta}[\mathrm{I}(g(\boldsymbol{\Theta}) \leq 0)]=\int_{\Omega_{\Theta}} \mathrm{I}[g(\boldsymbol{\theta}) \leq 0] \pi_{\boldsymbol{\Theta}}(\boldsymbol{\theta}) \mathrm{d} \boldsymbol{\theta},
$$

where the indicator function I equals 1 on the failure domain $\left\{\boldsymbol{\theta} \in \Omega_{\boldsymbol{\theta}}: g(\boldsymbol{\Theta}) \leq 0\right\}$ and 0 on its complement and $\pi_{\Theta}$ is the joint probability density of $\boldsymbol{\Theta}$.

Standard Monte Carlo methods fail to efficiently solve Eq. (1) if $\mathbb{P}(F)$ is very small, which is typically the case for failure probabilities. Methods which are specifically designed to solve Eq. (1) for very small $\mathbb{P}(F)$ are referred to as structural reliability methods (SRM). SRM can be categorized into approximation (e.g. FORM, SORM [2]) and sampling methods (e.g. importance sampling [3, 4], line sampling [5], subset simulation [6] and sequential importance sampling [7, 8]), both of which can be combined with surrogate modelling (e.g. $[9,10])$.

To account for different types of uncertainties (i.e. multi-uncertainty), we consider a segmentation of the 
random input vector in two disjunct subsets $\boldsymbol{\Theta}=\left[\boldsymbol{\Theta}_{A}, \boldsymbol{\Theta}_{B}\right]^{T}$. The variables $\boldsymbol{\Theta}_{B}$ can be interpreted as epistemic (sometimes also termed reducible) and the variables in $\boldsymbol{\Theta}_{A}$ as aleatory (irreducible). Irrespective of the interpretation of $\boldsymbol{\Theta}_{B}$, we aim at explicitly quantifying its influence on $\mathbb{P}(F)$. This is e.g. useful to identify those inputs in $\boldsymbol{\Theta}_{B}$ whose uncertainty should be reduced by means of additional data in order to increase the accuracy of the reliability analysis.

Figure 1 illustrates our framework for reliability analysis and updating in the presence of multi-uncertainty. To obtain information on the potential influence of each component of $\boldsymbol{\Theta}_{B}$ on the reliability analysis, we consider the probability of failure conditional on $\boldsymbol{\Theta}_{B}[11]$ :

$$
\begin{aligned}
P_{F}\left(\boldsymbol{\theta}_{B}\right) & =\mathbb{P}\left(F \mid \boldsymbol{\Theta}_{B}=\boldsymbol{\theta}_{B}\right) \\
& =\mathbb{E}_{\boldsymbol{\Theta}_{A}}\left[\mathrm{I}\left(g\left(\boldsymbol{\Theta}_{A}, \boldsymbol{\Theta}_{B}\right) \leq 0\right) \mid \boldsymbol{\Theta}_{B}=\boldsymbol{\theta}_{B}\right] \\
& =\int_{\Omega_{\Theta_{A}}} \mathrm{I}\left[g\left(\boldsymbol{\theta}_{A}, \boldsymbol{\theta}_{B}\right) \leq 0\right] f_{\boldsymbol{\Theta}_{A} \mid \boldsymbol{\Theta}_{B}}\left(\boldsymbol{\theta}_{A} \mid \boldsymbol{\theta}_{B}\right) \mathrm{d} \boldsymbol{\theta}_{A} .
\end{aligned}
$$

Note, that $P_{F}\left(\boldsymbol{\theta}_{B}\right)$ is a scalar function of the outcome of a random vector and hence is itself a random variable. A similar way of handling uncertainty separation is presented under the term 'second-order probability' in the program package Dakota [12] and is also discussed by other authors [11, 13].

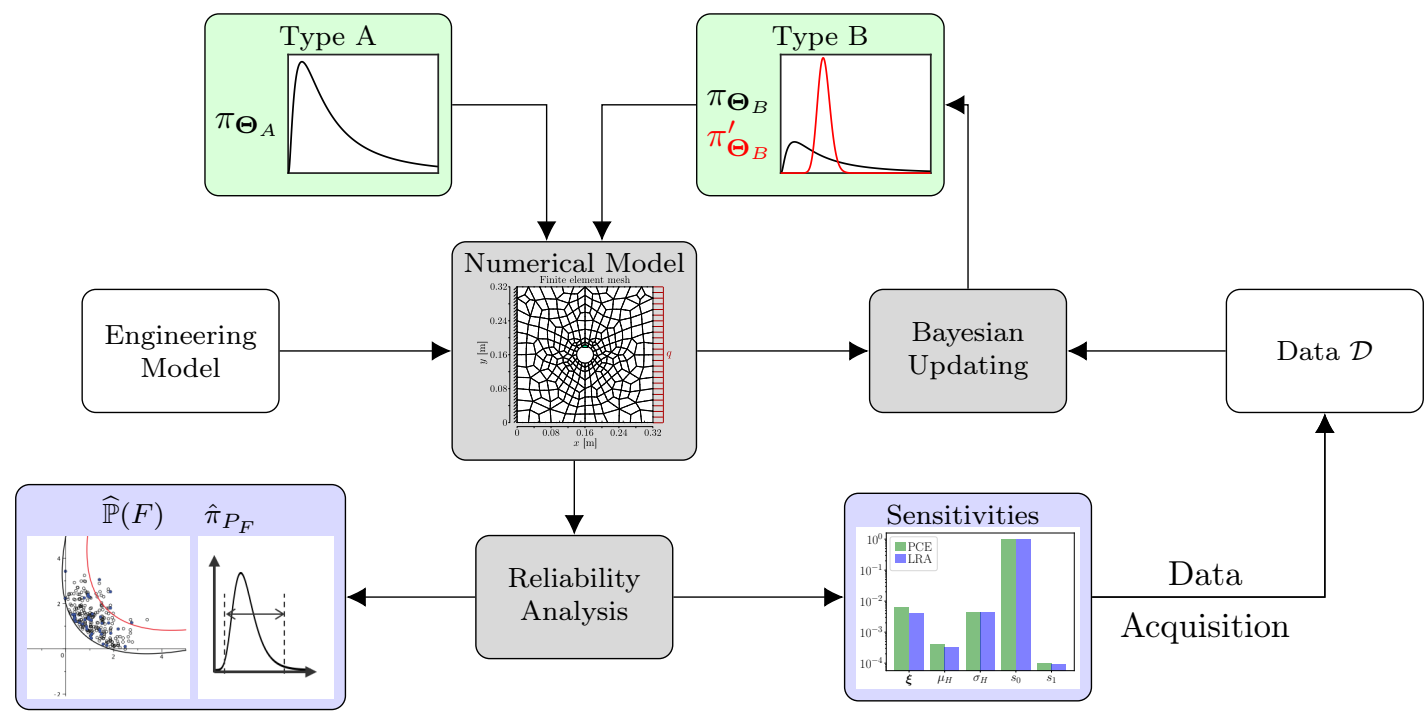

Figure 1: Framework for reliability sensitivity analysis in the presence of multi-uncertainty. The model $\mathcal{Y}$ is subject to irreducible $\left(\Theta_{A}\right)$ and reducible $\left(\Theta_{B}\right)$ probabilistic inputs. Estimates of the distribution of the probability of failure conditional on $\Theta_{B}, \hat{\pi}_{P_{F}}$, may be obtained through repeated reliability analyses fixed at different $\boldsymbol{\theta}_{B}$. Thus, $\hat{\pi}_{P_{F}}$ is subject to change when updating components of $\Theta_{B}$. The proposed reliability sensitivities quantify the obtainable variability reduction in $P_{F}$ resulting from uncertainty reduction (e.g. through Bayesian updating) in the components of $\boldsymbol{\Theta}_{B}$. In this way, they allow for an informed selection of data acquisition measures.

In the literature, a variety of metrics for quantifying sensitivities with respect to different quantities of interest can be found. They can be grouped according to scope (local [14] vs. global importance), momentdependence (e.g. variance-based $[15,16,17,18]$ vs. distribution-based [19, 20]) and considered inputs (deterministic parameters [21] vs. random variables [15, 16, 17, 18]). A good overview over global sensitivity measures is given in [22] and [23].

Most often, these measures are computed for the outcome of the numerical model. In contrast, reliabilityoriented sensitivity analysis is concerned with determining the sensitivity of the output of a reliability 
analysis to the model inputs. Many approaches dedicated to reliability-oriented sensitivities are concerned with determining local derivative-based sensitivity measures for deterministic model parameters in the limitstate function (LSF) [21] or deterministic distribution parameters of the input [24, 25, 26, 13]. Alternatively, [27] computes failure probability histograms conditional on design parameters (LSF or distribution parameters) by artificially consdering them as random variables. These histograms can be used to compute global sensitivity measures for the parameters. When using approximative reliability methods like FORM/SORM, reliability sensitivities are obtained from the analysis in form of the component (or $\alpha$-) values of the design point (or most probable point of failure) [2, 28]. These can be interpreted as global, variance-based sensitivity indices of the first-(FORM) and second-(SORM) order Taylor expansions of $g$ around the design point [28] in standard-normal space. Recently, [29] extended this idea to account for a mixture of several significant points and regions along the hypersurface $g=0$ by means of a Gaussian mixture (GM) model through defining a participation factor for each of the regional design point contributions of the GM components. [20] compute variance-based sensitivities for the indicator function $I(g \leq 0)$ and show, that this is equivalent to computing the moment-independent sensitivity measure proposed in [19] for the probability of failure. They use a surrogate model to relax the computational cost induced by the sampling-based sensitivity computation approach. Along the same lines, [30] also use a surrogate (Kriging) modelling approach when computing variance-based sensitivities for the probability of failure conditional on uncertain distribution parameters of the input variables. [31] compute variance-based sensitivity indices for a probability of failure conditional on distribution parameters using importance sampling and the FAST algorithm for estimating the sensitivity indices. Alternative sensitivity measures for rare event probabilities include the use of quantiles [32] or perturbation of input densities [33] to globally quantify influence of model inputs on rare event probabilities.

In this contribution, we introduce a reliability-oriented sensitivity measure that is based on the variance decomposition of the logarithm of the conditional probability of failure, defined in Eq. (2). The proposed measure enables the quantification of the influence of the type B (reducible inputs), which may consist of either input variables or uncertain distribution parameters thereof, on the magnitude of the probability of failure (Figure 1). To efficiently compute the proposed reliability sensitivities, we introduce a 2-level surrogate based approach. In the first level, a surrogate of the model response is constructed and is further used to compute the probability of failure conditional on samples of the type B-variables. The resulting conditional probability estimates are further used to construct a polynomial basis surrogate model of the logarithm of the conditional probability of failure. The coefficients of the level-2 surrogate can be post-processed to compute an estimate of the sought sensitivity indices.

The structure of the paper is as follows: In Section 2, we discuss the basics of global sensitivity analysis and introduce the new reliability sensitivity index. In Section 3, we present polynomial basis surrogate modelling (polynomial chaos expansions (PCEs) and low-rank approximations (LRAs)) and their relation to global sensitivity measures. Section 4 outlines a two-level framework for the surrogate-driven estimation of the novel reliability sensitivities, which is subsequently tested on two numerical examples in Section 5 . The studied examples consist of a 12-dimensional elastic truss structure and a 87-dimensional wind turbine monopile foundation in plastically behaving soil. Section 6 contains a discussion of the presented method and an outlook on possible extensions and improvements of the introduced framework.

\section{Global Reliability Sensitivity Analysis}

The sensitivity measures ptoposed here for reliability belong to the realm of variance-based sensitivity methods. After a brief introduction of the underlying idea of variance-based sensitivity indices for arbitrary quantities of interest, we move on to their application to reliability. We discuss the computational cost of these newly introduced indices when estimated with classical Monte Carlo-based (MC) approaches and thereby motivate a surrogate-driven computational approach, which is discussed in the next section. 


\subsection{Variance-Based Sensitivity Analysis}

Consider a $d$-dimensional standard uniform random vector $\boldsymbol{U} \sim \mathcal{U}_{d}(0,1)$, representing the input uncertainty of some model, and an output quantity of interest (QOI) $Q=f(\boldsymbol{U})$, with $f:[0,1]^{d} \rightarrow \mathbb{R}$.

Variance-based sensitivity indices rely on estimating the contribution of a single (first-order index) or a combination of the inputs $\boldsymbol{U}$ ( $n$-th order index, $n$ being the number of variables considered in the combination) to the output variance of the QOI. These fractions can be found by projecting the QOI on an orthogonal functional basis, which leads to a unique representation of $f$, namely the Sobol'-Hoeffding decomposition [15]

$$
f(\boldsymbol{U})=f_{0}+\sum_{i=1}^{d} f_{i}\left(U_{i}\right)+\sum_{i=1}^{d} \sum_{j=i+1}^{d} f_{i j}\left(U_{i}, U_{j}\right)+\cdots+f_{12 \ldots d}(\boldsymbol{U}) .
$$

Here, all basis functions have zero mean except for $f_{0}=\mathbb{E}[Q]$. If $Q$ depends on pairwise independent inputs with arbitrary distribution with known marginal CDFs, $F_{\Theta_{i}}$, an isoprobabilistic transformation can be used to generalize Eq. (3). This transformation is given by $T: \Theta_{i} \rightarrow F_{\Theta_{i}}\left(\Theta_{i}\right), 1 \leq i \leq d$, and the transformed decomposition is obtained by setting $\boldsymbol{U}=T(\mathbf{\Theta})$ in Eq. (3). By virtue of the orthogonality property, the variance of $f$ is merely the sum of the partial variances of all the basis functions. The partial variance corresponding to a set of inputs indicated by the index set $\mathcal{A} \subseteq\{1,2, \ldots, d\}$ is found as the variance of $f_{\mathcal{A}}$. The corresponding Sobol' index is then defined by the ratio

$$
S_{Q, \mathcal{A}}=\frac{\mathbb{V}\left[f_{\mathcal{A}}\right]}{\mathbb{V}[f]} .
$$

Note, that the Sobol' index measures the variance fraction that originates from a particular combination of variables exclusively without considering interaction of $\boldsymbol{\Theta}_{\mathcal{A}}$ with $\boldsymbol{\Theta}_{\sim \mathcal{A}}$, where $\sim$ denotes the complement of $\mathcal{A}$ over the total set $\{1,2, \ldots, d\}$. [16] defined the total-effect indices, which measure the partial variances of all basis functions including $\boldsymbol{\Theta}_{\mathcal{A}}$ :

$$
S_{Q, \mathcal{A}}^{T}=\frac{\sum_{\mathcal{I} \supseteq \mathcal{A}} \mathbb{V}\left[f_{\mathcal{I}}\right]}{\mathbb{V}[f]} .
$$

Alternatively, both Sobol' and total-effect indices can be cast in terms of variances of conditional expectations as [23]:

$$
\begin{aligned}
S_{Q, \mathcal{A}} & =\frac{\mathbb{V}_{\boldsymbol{\Theta}_{\mathcal{A}}}\left[\mathbb{E}_{\boldsymbol{\Theta}_{\sim \mathcal{A}}}\left[Q \mid \boldsymbol{\Theta}_{\mathcal{A}}\right]\right]}{\mathbb{V}[Q]}, \\
S_{Q, i}^{T} & =\frac{\mathbb{E}_{\boldsymbol{\Theta}_{\sim \mathcal{A}}}\left[\mathbb{V}_{\boldsymbol{\Theta}_{\mathcal{A}}}\left[Q \mid \boldsymbol{\Theta}_{\sim \mathcal{A}}\right]\right]}{\mathbb{V}[Q]} \\
& =1-\frac{\mathbb{V}_{\boldsymbol{\Theta}_{\sim \mathcal{A}}}\left[\mathbb{E}_{\boldsymbol{\Theta}_{\mathcal{A}}}\left[Q \mid \boldsymbol{\Theta}_{\sim \mathcal{A}}\right]\right]}{\mathbb{V}[Q]} .
\end{aligned}
$$

\subsection{The proposed reliability sensitivity indices}

In order to define variance-based sensitivities related to the reliabilty of a system one has to choose $Q$ appropriately. In reliability analysis, the quantity of interest is the failure event $F$ and the associated probability of failure. Since $F$ is defined via the indicator function of the failure domain, [20] propose to compute importance rankings through the variance decomposition of the indicator function $\mathrm{I}(g \leq 0)$. They do so by means of a surrogate modelling technique to avoid the slow convergence of standard MC-estimators in cases where the QOI is a rare event.

Within the multi-uncertainty setting, we propose to perform the variance decomposition of the conditional probability of failure defined in Eq. (2), i.e. More precisely, we propose using

$$
Q=\log P_{F}\left(\boldsymbol{\Theta}_{B}\right),
$$


where log indicates the logarithm to the base 10. This can be understood as a measure for the magnitude of the conditional probability of failure. In this way, we focus the sensitivity analysis on possibly substantial/magnitude-altering changes in the estimate of $P\left(\boldsymbol{\Theta}_{B}\right)$. Note that the concepts introduced in the following can be generalized to any quantity of interest that can be cast in terms of the expectation of a functional (e.g. any moment of $Y$ ). Consider now an arbitrary subset of $\boldsymbol{\Theta}_{B}$ which is denoted by $\boldsymbol{\Theta}_{\mathcal{B}}$ and its complement $\boldsymbol{\Theta}_{\sim \mathcal{B}}$ such that $\boldsymbol{\Theta}_{B}=\left\{\boldsymbol{\Theta}_{\mathcal{B}}, \boldsymbol{\Theta}_{\sim \mathcal{B}}\right\}$. The novel sensitivity indices for the variable subset $\boldsymbol{\Theta}_{\mathcal{B}}$ are given by

$$
\begin{aligned}
S_{\log P_{F}, \mathcal{B}} & =\frac{\mathbb{V}_{\boldsymbol{\Theta}_{\mathcal{B}}}\left[\mathbb{E}_{\boldsymbol{\Theta} \sim \mathcal{B}}\left[\log P_{F} \mid \boldsymbol{\Theta}_{\mathcal{B}}\right]\right]}{\mathbb{V}\left[\log P_{F}\right]}, \\
S_{\log P_{F}, \mathcal{B}}^{T} & =1-\frac{\mathbb{V}_{\boldsymbol{\Theta}_{\sim \mathcal{B}}}\left[\mathbb{E}_{\boldsymbol{\Theta}_{\mathcal{B}}}\left[\log P_{F} \mid \boldsymbol{\Theta}_{\sim \mathcal{B}}\right]\right]}{\mathbb{V}\left[\log P_{F}\right]} .
\end{aligned}
$$

Substituting Eq. (2) in the above, we arrive at the following expressions for the novel sensitivity indices:

$$
\begin{aligned}
S_{\log P_{F}, \mathcal{B}} & =\frac{\mathbb{V}_{\boldsymbol{\Theta}_{\mathcal{B}}}\left[\mathbb{E}_{\boldsymbol{\Theta}_{\sim \mathcal{B}}}\left[\log \left\{\mathbb{E}_{\boldsymbol{\Theta}_{A}}\left[\mathrm{I}(g \leq 0) \mid \boldsymbol{\Theta}_{B}\right]\right\} \mid \boldsymbol{\Theta}_{\mathcal{B}}\right]\right]}{\mathbb{V}_{\boldsymbol{\Theta}_{B}}\left[\log \left\{\mathbb{E}_{\boldsymbol{\Theta}_{A}}\left[\mathrm{I}(g \leq 0) \mid \boldsymbol{\Theta}_{B}\right]\right\}\right]} \\
S_{\log P_{F}, \mathcal{B}}^{T} & =1-\frac{\mathbb{V}_{\boldsymbol{\Theta}_{\sim \mathcal{B}}}\left[\mathbb{E}_{\boldsymbol{\Theta}_{\mathcal{B}}}\left[\log \left\{\mathbb{E}_{\boldsymbol{\Theta}_{A}}\left[\mathrm{I}(g \leq 0) \mid \boldsymbol{\Theta}_{B}\right]\right\} \mid \boldsymbol{\Theta}_{\sim \mathcal{B}}\right]\right]}{\mathbb{V}_{\boldsymbol{\Theta}_{B}}\left[\log \left\{\mathbb{E}_{\boldsymbol{\Theta}_{A}}\left[\mathrm{I}(g \leq 0) \mid \boldsymbol{\Theta}_{B}\right]\right\}\right]} .
\end{aligned}
$$

While these expressions appear cumbersome, they exhibit key features of the new indices:

1. The variance decomposition of the total variance contributed by $\boldsymbol{\Theta}_{B}$ rather than $\boldsymbol{\Theta}$ is performed, which is reflected by the normalizing constants in Eqs. (10) \& (11).

2. Due to the expectation $\mathbb{E}_{\Theta_{A}}$, the employed QOI is smooth on $\Omega_{\Theta_{B}}$. In particular, it is non-binary as opposed to the QOI underlying the indices proposed by [20].

3. The log-transformation of the conditional probability of failure focusses the reliability analysis on the probability of failure magnitude. From a numerical perspective, this is beneficial due to the additional smoothing of the the quantity of interest.

Remarkably, when decomposing the conditional probability of failure directly instead of its log-transformation, the Sobol' indices of the indicator function are recovered up to a factor (see Appendix A). In the following section, we discuss common problems when tackling reliability sensitivities with sampling methods. Thereafter, in Section 3, we introduce the means to circumvent sampling almost entirely in the computation of the novel sensitivity indices.

\subsection{Monte-Carlo estimators}

[17] and [18] provide Monte Carlo-estimators for the expressions in Eqs. (6). Based on a set of $n_{s} d$ dimensional $\Theta$-samples, $n_{s} \cdot(d+2) / 2$ model evaluations are necessary to compute them, where $n_{s}$ is the samples size required by the analysis. A reasonable estimate for $n_{s}$ is the number of samples required for the MC-estimation of $\mathbb{E}[Q]$ at a prescribed accuracy. These estimators may be intractable if a model evaluation is computationally expensive, $d$ is large or $Q$ is given by a failure event with small associated probability of failure. In the last case, intractability arises from the required amount of samples (i.e. evaluations of the $g$-function) to accurately estimate $\mathbb{E}[Q]$. It is $\approx 100 / \mathbb{P}(F)$ when the allowed coefficient of variation of the estimator is $10 \%$. Therefore, when typically $\mathbb{P}(F)$ is very small, $n_{s}$ becomes prohibitively large.

For the novel sensitivity indices, the computational burden would amount to a multiple of what is needed for the computation of sensitivity indices of the indicator function of $g$. This is due to the need to solve Eq. (2) $n_{s} \cdot(d+2) / 2$ times, which may in turn require many $g$-evaluations per solution. Conversely, computing the indices associated with $Q=\mathrm{I}(g \leq 0)$ requires a single $g$-evaluation at each sample to determine whether $g \leq 0$. Therefore, the computational effort scales approximately as the average number of $g$-calls necessary to solve Eq. (2) at constant $n_{s}$ (although, at convergence, $n_{s}$ would likely be considerably smaller compared to the case where $Q=\mathrm{I}(g \leq 0))$. However, the smoothness in our choice of $Q$ is key to an entirely 
surrogate-driven sensitivity computation, which facilitates the use of only a small fraction of the samples required in the sampling-based procedure. Two types of surrogate models have been tested and are detailed in the subsequent section.

\section{Polynomial Basis Surrogate Modelling}

Let $\Theta$ be a random vector on the outcome space $\mathbb{R}^{d}$ with joint $\operatorname{CDF} F_{\Theta}$ whose elements are mutually independent and $Y=\mathcal{Y}(\boldsymbol{\Theta})$, with $\mathcal{Y}: \mathbb{R}^{d} \rightarrow \mathbb{R}$. If $Y$ has finite mean-square, i.e. $\mathbb{E}_{\boldsymbol{\Theta}}\left[\mathcal{Y}(\boldsymbol{\Theta})^{2}\right]<\infty$, then the function $\mathcal{Y}$ belongs in a Hilbert space $\mathcal{H}$ on which an inner product of any two functions $g, h \in \mathcal{H}$ is defined as

$$
\langle g(\boldsymbol{\theta}), h(\boldsymbol{\theta})\rangle_{\mathcal{H}}=\mathbb{E}_{\boldsymbol{\Theta}}[g(\boldsymbol{\Theta}) h(\boldsymbol{\Theta})]=\int_{\mathbb{R}^{d}} g(\boldsymbol{\theta}) h(\boldsymbol{\theta}) \pi_{\boldsymbol{\Theta}}(\boldsymbol{\theta}) \mathrm{d} \boldsymbol{\theta},
$$

where $\pi_{\boldsymbol{\Theta}}(\boldsymbol{\theta})$ is the joint probability density function (PDF) of $\boldsymbol{\Theta} . g$ and $h$ are orthogonal if

$$
\langle g(\boldsymbol{\theta}), h(\boldsymbol{\theta})\rangle_{\mathcal{H}}=\mathbb{E}_{\boldsymbol{\Theta}}[g(\boldsymbol{\Theta}) h(\boldsymbol{\Theta})]=0 .
$$

Note, that if $g$ and $h$ can be written as products of univariate functions of the components of $\boldsymbol{\Theta}$, the following holds:

$$
\langle g(\boldsymbol{\theta}), h(\boldsymbol{\theta})\rangle_{\mathcal{H}}=\prod_{i=1}^{d} \mathbb{E}_{\Theta_{i}}\left[g_{i}\left(\Theta_{i}\right) h_{i}\left(\Theta_{i}\right)\right] .
$$

Given a complete and orthonormal basis of $\mathcal{H},\left\{h_{i}(\boldsymbol{\theta}), i \in \mathbb{N}\right\}, Y$ may be expressed as a linear combination of the basis functions:

$$
Y=\mathcal{Y}(\mathbf{\Theta})=\sum_{i=0}^{\infty} a_{i} h_{i}(\mathbf{\Theta})
$$

Then, since $\mathcal{Y} \in \mathcal{H}$, the approximation

$$
\widehat{Y}=\widehat{\mathcal{Y}}(\boldsymbol{\Theta})=\sum_{i=0}^{p} a_{i} h_{i}(\mathbf{\Theta})
$$

asymptotically $(p \rightarrow \infty)$ converges to $Y$ in the mean-square sense. For $d=1$, a possible choice of basis functions related to certain standard distribution types of $\pi_{\Theta}$ are known polynomial families $\left\{\psi_{i}(\theta), i=\right.$ $0, \ldots, p\}$, which are identified by means of the Askey scheme [34]. This lays the foundation for both PCEs and LRAs. They differ with respect to how the multi-dimensional base polynomials are defined and how the expansion coefficients $a_{i}$ are determined. For $d>1$, due to Eq. (14), multi-dimensional basis polynomials $\Psi_{k}$ can be easily constructed as products of the one-dimensional canonical polynomials $\psi_{k}^{(i)}$.

\subsection{Polynomial Chaos Expansions}

Given the polynomial family of the $i$-th input $\theta_{i}$ up to $p_{i}$-th order $\left\{\psi_{j}^{(i)}\left(\theta_{i}\right), j=0, \ldots, p_{i}\right\}$, the $j$-th multi-dimensional basis function reads

$$
\Psi_{j}(\boldsymbol{\theta})=\prod_{i=1}^{d} \psi_{\alpha_{j i}}^{(i)}\left(\theta_{i}\right)
$$

where $\boldsymbol{\alpha}$ contains all combinations of $d$-dimensional index sets each assigning a polynomial order to each input $\theta_{i}$ such that the total polynomial order $\left|\boldsymbol{\alpha}_{j}\right|=\sum_{i=1}^{d} \alpha_{j i} \leq p, 0 \leq j \leq P-1$. The number of basis functions $P$ is given by

$$
P=\left(\begin{array}{c}
d+p \\
p
\end{array}\right)
$$

and the PCE format reads

$$
\widehat{\mathcal{Y}}^{P C E}(\boldsymbol{\Theta})=\sum_{j=0}^{P-1} a_{j} \prod_{i=1}^{d} \psi_{\alpha_{j i}}^{(i)}\left(\Theta_{i}\right) .
$$


The coefficients $\boldsymbol{a}$ are identified through a projection of $\mathcal{Y}$ onto the space spanned by $\left\{\Psi_{j}, j=0, \ldots, P-1\right\}$. In this work, we evaluate $\boldsymbol{a}$ using an ordinary least-squares (OLS) approach, which approximates the projection of $\mathcal{Y}$ onto the PCE basis [35]. Consider a set of $n_{s}$ samples $\boldsymbol{X}$ of the input random vector $\boldsymbol{\Theta}$ called the experimental design and corresponding $\mathcal{Y}$-evaluations $\boldsymbol{Y}$, where $\boldsymbol{X} \in \mathbb{R}^{n_{s} \times d}$ and $\boldsymbol{Y} \in \mathbb{R}^{n_{s} \times 1}$. We call the collection $\mathcal{E}=\{\boldsymbol{X}, \boldsymbol{Y}\}$ the training set. The data matrix $\boldsymbol{\Psi}$ collects the evaluation of each of the multi-dimensional basis polynomials at each point in $\boldsymbol{X}$

$$
\boldsymbol{\Psi}=\Psi_{j}\left(\boldsymbol{x}^{(i)}\right), 1 \leq i \leq n_{s}, 1 \leq j \leq P,
$$

where $\boldsymbol{x}^{(i)}$ is the $i$-th row of $\boldsymbol{X}$. The vector of all $P$ PCE-coefficients $\boldsymbol{a}$ is then obtained by regressing $\boldsymbol{Y}$ on $\Psi$ which gives

$$
\boldsymbol{a}=\left(\boldsymbol{\Psi}^{T} \boldsymbol{\Psi}\right)^{-1} \boldsymbol{\Psi}^{T} \boldsymbol{Y} .
$$

Equation (18) indicates a fast growth of the OLS problem size with increasing dimension $d$. This motivates the use of sparse PCE methods, which are also applied in this work. Sparse PCE reduces $P$ by penalizing the number of terms in the PCE through solving a regularized least-squares problem [36]. In this way, the method elicits a minimal number of basis functions such as to best explain the output variance.

\subsection{Canonical Decomposition}

Low-rank approximations have been introduced originally to represent high-dimensional tensors by means of lower-dimensional tensors [37]. A specific format of such approximations are canonical decompositions, in which tensors are approximated by means of a linear combination of products of one-dimensional tensors [38]. The idea extends to continuous spaces where a multivariate function is approximated by a linear combination of products of univariate functions:

$$
\widehat{\mathcal{Y}}^{L R A}(\boldsymbol{\Theta})=\sum_{j=1}^{r} a_{j} \prod_{i=1}^{d} \sum_{k=1}^{p_{i}} z_{i j k} \psi_{k}^{(i)}\left(\Theta_{i}\right) .
$$

Therein, an additional set of coefficients $z$ appears, which can be efficiently determined by solving reduced, univariate least squares problems over the directions $i=1, \ldots, d$ repeatedly (while keeping all remaining directions constant in each step; this is often referred to as alternating least squares). In a second step, the coefficients $\boldsymbol{a}$ are determined via OLS. A detailed description of the procedure is given in [39] and [40]. The number of cofficients in the canonical decomposition is $\mathcal{O}((p+1) \cdot d \cdot r)$ assuming a constant polynomial degree $p$ in all dimensions. This linearity in $d$ is remarkable and explains the advantage this format offers over classical PCE where the coefficients grow factorially with the dimension as described in Eq. (18).

\subsection{Surrogate-Based sensitivity indices}

Both PCEs and LRAs can be used to infer first-order and total sensitivity indices directly from the computed model coefficients. Rather than searching estimates of the expressions in Eqs. (6), the similarity of the underlying orthogonal Sobol' decomposition in Eq. (3) with Eqs. (19) and (22) is exploited and the expressions (4) and (5) can be computed directly. [41] showed that the Sobol' decomposition of the PCE is readily obtained by collecting any multi-dimensional orthogonal polynomials depending on identical variable subsets $\Theta_{\mathcal{A}}$ into $f_{\mathcal{A}}\left(\boldsymbol{\Theta}_{\mathcal{A}}\right)$. Therefore, computing the partial variance of the PCE model associated with a subset of variables $\boldsymbol{\Theta}_{\mathcal{A}}$ amounts to summing the squared coefficients of the respective multi-dimensional basis polynomials in which the elements of $\boldsymbol{\Theta}_{\mathcal{A}}$ occur (exclusively for Sobol' indices and collectively for total indices). The same concept can be applied to LRAs even though the compressed format (product) renders the evaluation somewhat more tedious. For a given subset of the input variables denoted by the index set $\mathcal{A}$, we define a boolean index vector $\mathcal{I}^{\mathcal{A}} \in\{0,1\}^{d}$ s.t. $\mathcal{I}_{i}^{\mathcal{A}}=0$ if $i \notin \mathcal{A}$ and $\mathcal{I}_{i}^{\mathcal{A}}=1$ if $i \in \mathcal{A}$. In the same way, we define such an index vector for the $j$-th row of $\boldsymbol{\alpha}_{j}$ s.t. $\mathcal{I}_{i}^{\boldsymbol{\alpha}_{j}}=0$ if $\boldsymbol{\alpha}_{i j}=0$ and $\mathcal{I}_{i}^{\boldsymbol{\alpha}_{j}}=1$ if $\boldsymbol{\alpha}_{i j}>0$. Then, the PCE-based sensitivity indices read [41]:

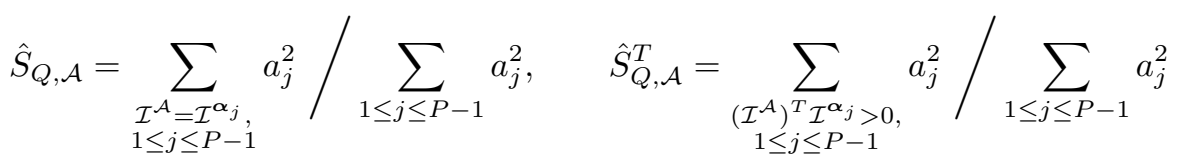


and the LRA-based indices [42]:

$$
\begin{aligned}
\hat{S}_{Q, \mathcal{A}}=\frac{\sum_{j=1}^{r} \sum_{j^{\prime}=1}^{r} a_{j} a_{j^{\prime}}\left(\prod_{i \notin \mathcal{A}} z_{i j, 0} z_{i j^{\prime}, 0}\right)\left(\prod_{i^{\prime} \in \mathcal{A}}\left(\sum_{k=1}^{p_{i}} z_{i^{\prime} j k} z_{i^{\prime} j^{\prime} k}\right)\right)-\sum_{j=1}^{r} \sum_{j^{\prime}=1}^{r} a_{j} a_{j^{\prime}}\left(\prod_{i=1}^{d}\left(\sum_{k=1}^{p_{i}} z_{i j k} z_{i j^{\prime} k}\right)\right)}{\sum_{j=1}^{r} \sum_{j^{\prime}=1}^{r} a_{j} a_{j^{\prime}}\left(\left(\prod_{i=1}^{d}\left(\sum_{k=1}^{p_{i}} z_{i j k} z_{i j^{\prime} k}\right)\right)-\left(\prod_{i=1}^{d} z_{i j, 0} z_{i j^{\prime}, 0}\right)\right)}, \\
\hat{S}_{Q, \mathcal{A}}^{T}=1-\frac{\sum_{j=1}^{r} \sum_{j^{\prime}=1}^{r} a_{j} a_{j^{\prime}}\left(\prod_{i \in \mathcal{A}} z_{i j, 0} z_{i j^{\prime}, 0}\right)\left(\prod_{i^{\prime} \notin \mathcal{A}}\left(\sum_{k=1}^{p_{i}} z_{i^{\prime} j k} z_{i^{\prime} j^{\prime} k}\right)\right)-\sum_{j=1}^{r} \sum_{j^{\prime}=1}^{r} a_{j} a_{j^{\prime}}\left(\prod_{i=1}^{d}\left(\sum_{k=1}^{p_{i}} z_{i j k} z_{i j^{\prime} k}\right)\right)}{\sum_{j=1}^{r} \sum_{j^{\prime}=1}^{r} a_{j} a_{j^{\prime}}\left(\left(\prod_{i=1}^{d}\left(\sum_{k=1}^{p_{i}} z_{i j k} z_{i j^{\prime} k}\right)\right)-\left(\prod_{i=1}^{d} z_{i j, 0} z_{i j^{\prime}, 0}\right)\right)} .
\end{aligned}
$$

\section{Conditional Surrogate-Based Reliability Sensitivities}

The computation of sensitivity indices via polynomial surrogates requires the QOI to be sufficiently smooth. In particular, any attempts to obtain surrogate-based indices of the indicator function of the failure domain $\mathrm{I}(g \leq 0)$ directly in such a manner must fail due to the discontinuity in $\mathrm{I}(g \leq 0)$. However, the log-transformed conditional probability of failure is continuous in the space of $\boldsymbol{\Theta}_{B}$ so that one may compute the proposed sensitivity indices with polynomial surrogates. To this end, we devise a two-level surrogate modelling procedure. Building a surrogate of $\log P_{F}$ (level 2) requires an experimental design that consists of samples of $\boldsymbol{\Theta}_{B}$ and the associated probabilities of failure given each of these samples. That is, one has to solve $n_{2}$ reliability problems, where $n_{2}$ is the experimental design size for the final surrogate. An auxiliary (level 1) surrogate is built for the actual model $\mathcal{Y}$, based on which the reliability computations can be conducted. This approach is referred to as global in the following. Alternatively, a local surrogate model may be constructed at each sample of $\boldsymbol{\Theta}_{B}$ for solving its associated reliability problem. The local surrogate has lower-dimensional input compared to the global surrogate because the input space is reduced from $\Omega_{\Theta}$ to $\Omega_{\Theta_{A}}$, and is therefore cheaper to determine; however, it has to be recomputed for each $\boldsymbol{\Theta}_{B}$-sample. This approach will be referred to as local. Prior to construction, an isoprobabilistic transformation to an independent standard-normal space is used, such that both PCEs and LRAs can be consistently constructed using the the orthogonal polynomial basis with respect to the standard normal probability measure, which is the Hermite polynomial basis [34].

\subsection{Level 1}

On the first level, the goal is to construct a surrogate model for the original model $\mathcal{Y}(\boldsymbol{\Theta})$, describing the engineering system.

\subsubsection{Global Approach}

In the global approach, we evaluate the model at the level 1-experimental design which yields the level 1-training set $\mathcal{E}_{1}=\left\{\boldsymbol{X}_{1}, \boldsymbol{Y}_{1}\right\}$, where $\boldsymbol{X}_{1} \in \mathbb{R}^{n_{1} \times d}$ is drawn from $\pi_{\Theta}$ and $\boldsymbol{Y}_{1}=\mathcal{Y}\left(\boldsymbol{X}_{1}\right) \in \mathbb{R}^{n_{1} \times 1}$ and $n_{1}$ is the number of points in $\mathcal{E}_{1}$. The overall number of original model evaluations is thereby limited to $n_{1}$ because any subsequent computations, namely the reliability analyses, will be run with the level 1-surrogate. In this level, any kind of surrogate modelling technique can be utilized to run the reliability analysis. However, the quality of the reliability sensitivity estimates mostly depends on the quality of the surrogate and the applied structural reliability method (SRM) in level 1. Thus, tuning the method in this component will yield the most substantial improvements in estimating the reliability indices. [9] introduced a Kriging-driven MonteCarlo sampling approach which enriches the Kriging experimental design according to a learning function that favors large model uncertainty close to the limit-state hypersurface $g=0$ and [43] applied a similar idea to PCEs. A recently introduced surrogate-driven sequential sampling approach for reliability analysis explores the failure domain sequentially and reconstructs a surrogate model at each intermediate step in the 
sampling procedure [44]. All of these approaches have been shown to substantially improve the unconditional reliability estimate.

\subsubsection{Local Approach}

For nonlinear models, $\Omega_{\Theta_{B}}$ may contain regions in which the conditional probability of failure becomes either very small or very large. Global surrogate methods may fail to reconstruct the model accurately in such regions and thereby introduce an error in the estimator of the proposed sensitivity indices. In such case, one may instead use cheap local surrogates which are reconstructed at each $\boldsymbol{\Theta}_{B}$-sample. At the $i$-th sample $\boldsymbol{\theta}_{B}^{(i)}$, the local surrogate $\widehat{\mathcal{Y}} \mid \boldsymbol{\theta}_{B}^{(i)}\left(\boldsymbol{\Theta}_{A}\right)$ is constructed based on a local training set $\mathcal{E}_{1}^{(i)}=\left\{\boldsymbol{X}_{1}^{(i)}, \boldsymbol{Y}_{1}^{(i)}\right\}$, where $\boldsymbol{X}_{1}^{(i)} \sim \pi_{\boldsymbol{\Theta}_{A}}$ and $\boldsymbol{Y}_{1}^{(i)}=\mathcal{Y}\left(\boldsymbol{X}_{1}^{(i)}, \boldsymbol{\theta}_{B}^{(i)}\right)$. The local approach is particularly suitable if $d_{B}=\operatorname{dim}\left(\boldsymbol{\Theta}_{B}\right)$ is large $\left(d_{B} / d \rightarrow 1\right)$, i.e. most variables are reducible, since then, the resulting conditional reliability problems are low-dimensional (they are posed on $\Omega_{\Theta_{A}}$, which has dimension $\left.d_{A}=\operatorname{dim}\left(\boldsymbol{\Theta}_{A}\right)=d-d_{B}\right)$. In such case, the local surrogates depend on a low-dimensional input such that they can be computed using small experimental designs. A more detailed discussion of computational cost depending on the variable splitting in $\boldsymbol{\Theta}_{A}$ and $\boldsymbol{\Theta}_{B}$ is given in Section 4.4 .

\section{Effective surrogate model dimension for input mixtures}

When considering both random inputs and uncertain parameters of these inputs at the same time, one can reduce the effective dimension of the experimental design over which to construct the level 1surrogate. Consider a random vector $\boldsymbol{\Theta}$, where $\Theta_{2}=M, \Theta_{3}=\Sigma$ are uncertain parameters of $\Theta_{1}$ which is a random variable. The probabilistic model of $\Theta_{1}$, through the conditional CDF $F_{\Theta_{1} \mid M, \Sigma}$, establishes an exact relationship between $\Theta_{1}, M$ and $\Sigma$. If $F_{\Theta_{1} \mid M, \Sigma}$ is invertible in $\Theta_{1}$, the following hierarchical sampling strategy yields a sample from $\Theta_{1}$ based on a sample $\boldsymbol{u}=\left[u_{1}, u_{2}, u_{3}\right]^{T}$ from the standard-uniform distribution.

$$
\begin{aligned}
m & =F_{M}^{-1}\left(u_{1}\right), \\
\sigma & \left.=F_{\Sigma}^{-1}\left(u_{2}\right)\right), \\
\theta_{1} & =F_{\Theta_{1} \mid M, \Sigma}^{-1}\left(u_{3} \mid m, \sigma\right)=F_{\Theta_{1} \mid M, \Sigma}^{-1}\left(u_{3} \mid F_{M}^{-1}\left(u_{1}\right), F_{\Sigma}^{-1}\left(u_{2}\right)\right) .
\end{aligned}
$$

Since the surrogate is constructed in standard-normal space, we use an isoprobabilistic transformation to compute the corresponding standard-normal sample $v_{1}$ :

$$
v_{1}=\Phi^{-1}\left[F_{\Theta_{1}}\left(\theta_{1}\right)\right]
$$

where $\Phi(\cdot)$ is the standard-normal CDF. Equations (26) \& (27) facilitate the construction of a surrogate model for the marginalized input space, which does not contain $M$ and $\Sigma$ anymore. In this way, we improve the surrogate accuracy through replacing what would have been an approximation of the interaction amongst $\Theta_{1}, M$ and $\Sigma$ with their exact relationship. Instead of marginalizing the input space, one may also work in the higher-dimensional space and obtain larger experimental designs at no additional computational cost by sampling $\left[\Theta_{1}, M, \Sigma\right]$ from iso- $\Theta_{1}$-surfaces, as done in [45]. Then, the PCE will still approximate the exact relationship amongst $\Theta_{1}, M$ and $\Sigma$, though at considerably better accuracy through the larger training set. The predictive CDF of $\Theta_{1}, F_{\Theta_{1}}$, can be computed via numerical integration, e.g. Monte-Carlo integration:

$$
\begin{aligned}
F_{\Theta_{1}}\left(\theta_{1}\right) & =\int_{-\infty}^{\infty} \int_{-\infty}^{\infty} F_{\Theta_{1} \mid M, \Sigma}\left(\theta_{1} \mid m, \sigma\right) \pi_{M}(m) \pi_{\Sigma}(\sigma) \mathrm{d} m \mathrm{~d} \sigma \\
& =\mathbb{E}\left[F_{\Theta_{1} \mid M, \Sigma}\left(\theta_{1} \mid M, \Sigma\right)\right] \\
& \approx \frac{1}{N} \sum_{i=1}^{N} F_{\Theta_{1} \mid M, \Sigma}\left(\theta_{1} \mid m^{i}, \sigma^{i}\right), \quad m^{i} \sim \pi_{M}, \quad \sigma^{i} \sim \pi_{\Sigma}
\end{aligned}
$$




\subsection{Level 2}

The level 2-surrogate $\widehat{\log P_{F}}$ is based on the training set $\mathcal{E}_{2}=\left\{\boldsymbol{X}_{2}, \boldsymbol{Y}_{2}\right\}$, where $\boldsymbol{X}_{2} \in \mathbb{R}^{n_{2} \times d_{B}}$ is drawn from $\pi_{\boldsymbol{\Theta}_{B}}, \boldsymbol{Y}_{2}=\log P_{F}\left(\boldsymbol{X}_{2}\right) \in \mathbb{R}^{n_{2} \times 1}$ and $n_{2}$ is the number of points in $\mathcal{E}_{2}$. In level 2, we focus on polynomial basis surrogates, such that no additional (not even surrogate-model-based) sampling is required to obtain the reliability sensitivities (Section 3). The level 2-surrogate model additionally provides a cheap and approximate method for updating probability-of-failure-densities. That is, upon obtaining posterior $\boldsymbol{\Theta}_{B^{-}}$ samples through Bayesian updating, the associated posterior failure density (and its mean, the predictive reliability estimate) can be computed through the analytical function $\widehat{\log P_{F}}\left(\boldsymbol{\Theta}_{B}\right)$ rather than solving a reliability problem for each of the posterior samples. The polynomial basis in $\log P_{F}$ is orthogonal with respect to the prior input joint density. Thus, coefficient-based postprocessing (moments, sensitivities) of the posterior conditional probability of failure, should be handled with great care.

\subsection{The Framework}

The analysis proceeds in the following way:

1. Elicit a variable subset of interest $\boldsymbol{\Theta}_{B}$. Obtain $n_{2} \boldsymbol{\Theta}_{B}$-samples, $\left\{\boldsymbol{\theta}_{B}^{(i)}\right\}_{1 \leq i \leq n_{2}}$ (e.g. based on latin hypercube sampling or a low-discrepancy sequence).

2. In case $\boldsymbol{\Theta}_{B}$ comprises a mixture of inputs and uncertain parameters thereof, marginalize the input space according to Eqs. (26), (27) and (28).

3. Select the global or local strategy for the level 1-surrogate.

\section{a) Global}

Sample $n_{1} \Theta$-samples and evaluate the model $\mathcal{Y}$ at these samples. Based on these, build the global level 1-surrogate over $\Omega_{\Theta}, \widehat{\mathcal{Y}}$.

For each $\Theta_{B}$-sample, use a structural reliability method and the global level 1-surrogate-based limitstate function $\widehat{g}$ to compute

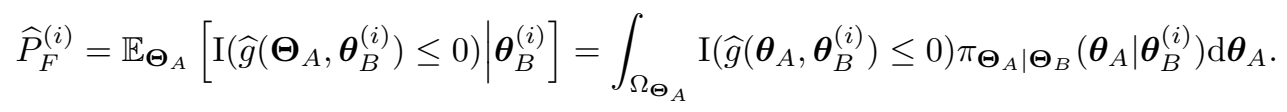

\section{b) Local}

For each $\boldsymbol{\Theta}_{B}$-sample, sample $n_{1} \Theta_{A}$-samples and evaluate the model $\mathcal{Y}$ at these samples. Based on these, build the local level 1-surrogate over $\Omega_{\Theta_{A}}$ conditional on $\boldsymbol{\theta}_{B}^{(i)}, \widehat{\mathcal{Y}} \mid \boldsymbol{\theta}_{B}^{(i)}$ and its associated local limit-state function $\widehat{g}_{i}\left(\boldsymbol{\theta}_{A}\right)$ to compute $\widehat{\mathcal{Y}} \mid \boldsymbol{\theta}_{B}^{(i)}$

$$
\widehat{P}_{F}^{(i)}=\mathbb{E}_{\boldsymbol{\Theta}_{A}}\left[\mathrm{I}\left(\widehat{g}_{i}\left(\boldsymbol{\theta}_{A}\right) \leq 0\right)\right]=\int_{\Omega_{\boldsymbol{\Theta}_{A}}} \mathrm{I}\left(\widehat{g}_{i}\left(\boldsymbol{\theta}_{A}\right) \leq 0\right) \pi_{\boldsymbol{\Theta}_{A} \mid \boldsymbol{\Theta}_{B}}\left(\boldsymbol{\theta}_{A} \mid \boldsymbol{\theta}_{B}^{(i)}\right) \mathrm{d} \boldsymbol{\theta}_{A} .
$$

4. From the set $\left\{\boldsymbol{\theta}_{B}^{(i)}, \log \left(\widehat{P}_{F}^{(i)}\right)\right\}_{i=1, \cdots, n_{2}}$, build the level 2-surrogate $\widehat{\log P_{F}}\left(\boldsymbol{\Theta}_{B}\right)$.

5. Obtain variance-based sensitivity indices of $\widehat{\log P}_{F}$ by means of the model coefficients.

The procedure outlined above is also sketched in Figures 2 (global) \& 3 (local).

\subsection{Computational cost}

Figure 4 shows the behaviour of computational cost in both levels when using either a global or a local or no surrogate strategy at all in level 1 . The computational cost is measured in terms of number of $\mathcal{Y}$ evaluations. To proceed, we make the assumption that the number of samples required to learn a surrogate model in input dimension $d$ is $n=2 \cdot(d+1)$. Note that this is a rather crude assumption and is accurate only for mildly nonlinear models $\mathcal{Y}$. The number of samples required in level $2, n_{2}=2\left(d_{B}+1\right)$, is the same in all three versions, though for the global surrogate model, this does not influence the computational cost. At fixed model dimension $d$, the global surrogate modelling costs are $n_{1, \text { global }}=n_{\text {global }}=2(d+1)$, 


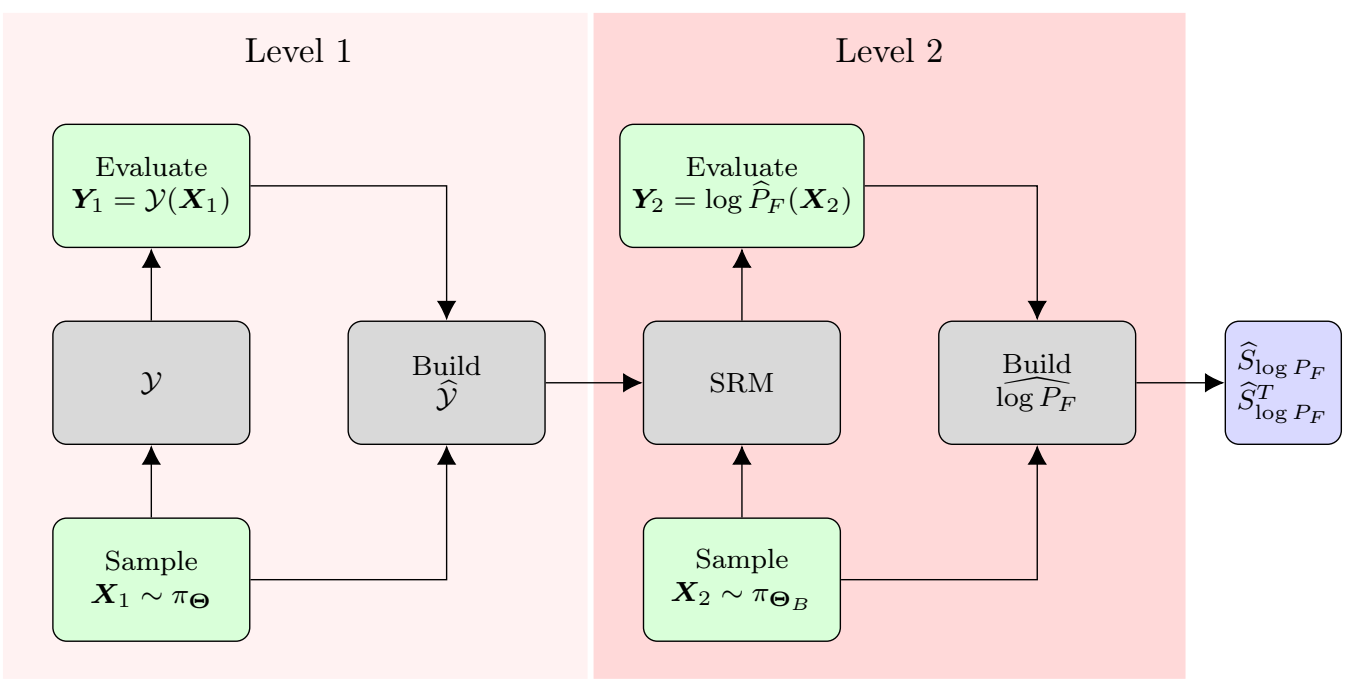

Figure 2: Flow diagram of 2-level surrogate-based conditional reliability sensitivities with global surrogate in level 1 .

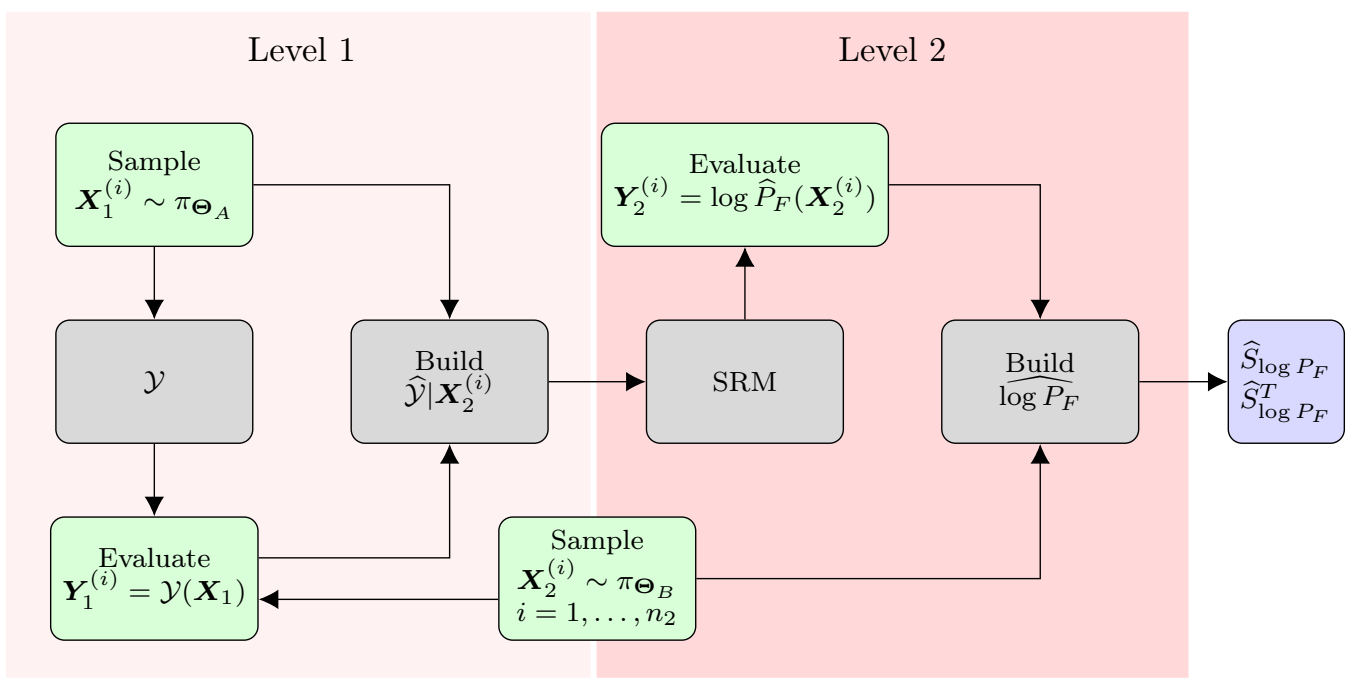

Figure 3: Flow diagram of 2-level surrogate-based conditional reliability sensitivities with local surrogates in level 1.

i.e. they are constant, depend only on level 1 and the total dimension and not on $d_{A}$ or $d_{B}$. For the local surrogate strategy, the cost in level 1 is given by $n_{1, \text { local }}=2\left(d_{A}+1\right)$ per $\boldsymbol{\Theta}_{B}$-sample. The total cost thus reads $n_{\text {local }}=n_{1, \text { local }} \cdot n_{2}$. When using no level 1-surrogate at all, $n_{2}$ reliability problems are solved using the original model $\mathcal{Y}$. In order to estimate the computational cost in the surrogate-free approach, the number of required $\mathcal{Y}$-evaluations to solve a $d_{A}$-dimensional reliability problem, $n_{1, \mathcal{Y}}$, has to be estimated. Therein, $d_{A}$ influences which method should be used to solve the problem. The curve representing this number in Figure 4 is obtained by fitting a polynomial model when assuming $\left[4,10,100,10^{4}\right]$ required $\mathcal{Y}$-evaluations to solve a $[1,2,10,100]$-dimensional reliability problem with constant target probability of failure at a prescribed accuracy using e.g. [Bisection, FORM, Importance Sampling, Subset Simulation]. The total cost in this approach is computed as $n_{\mathcal{Y}}=n_{1, \mathcal{Y}} \cdot n_{2}$.

Figure 4 (right-hand side) shows, that both the local surrogate and surrogate-free strategy yield the highest overall computational cost when $d_{B}$ and $d_{A}$ have similar size, i.e. neither reducible nor irreducible uncertainties dominate the model input. This is due to two counter-acting effects: On the one hand, as the 

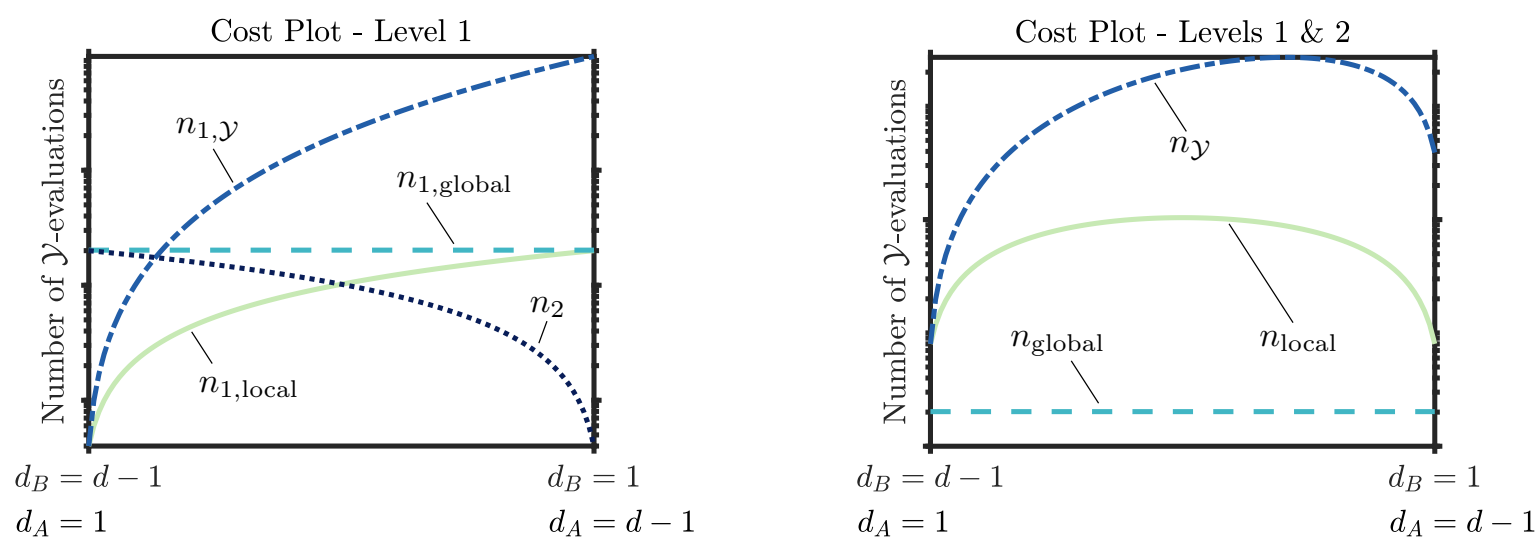

Figure 4: Comparison of global (global) vs. local (local) vs. no $(\mathcal{Y})$ surrogate strategy in level 1 in terms of computational cost (number of $\mathcal{Y}$-calls) over reducible space dimension $d_{B}$. Left: cost per level, right: total cost.

effective local surrogate model dimension equals $d_{A}$, the local strategy requires less samples per surrogate when $d_{A}$ is small. Similarly, if not using a surrogate at all in level 1, the reliability analysis performed with the original model will require less model evaluations due to the decreasing $d_{A}$. On the other hand, decreasing $d_{A}$ implies an increasing reducible space dimension $d_{B}$. Thus, in order to build accurate level 2-surrogates, $n_{2}$ needs to increase as well implying a multiplication of the overall number of required local surrogates/original-model-based reliability analyses. Here, the global surrogate model seems to be the most efficient. This, however, is only true when $\mathcal{Y}$ is mildly nonlinear. For models that exhibit stronger nonlinear behaviour, preserving the accuracy of the global model may require more model evaluations than the local strategy. In many application cases, the local strategy could be operated efficiently as the number of reducible inputs is considerably lower than the number of irreducible inputs $d_{B}<<d_{A}$.

\section{Numerical examples}

The novel reliability sensitivity indices are investigated and demonstrated through two applications, namely an elastic truss of moderate dimensionality (12 dimensions) and a monopile foundation in plastic soil involving a random field model (87 dimensions). Both examples feature both independent input variables as well as uncertain parameters thereof. The level 1-surrogate is constructed with LRAs which yield consistently smaller global and conditional (on failure samples) global errors. This is in accordance with the findings of [40].

\subsection{Elastic truss}

The truss structure (Figure 5) consists of 13 rods, where horizontal and diagonal rods have log-normally distributed cross-sections $A_{1}, A_{2}$ and Young's moduli $E_{1}, E_{2}$, respectively. The truss sustains 6 vertical point loads $P_{1}-P_{6}$ which are modelled as Gumbel-distributed [46, 42]. [47] presents results for an analysis of the original elastic truss with the proposed sensitivity framework. Here an extension of the truss model featuring hyperparameters is discussed. Namely, the parameters $a_{P}, b_{P}$ of the load Gumbel distribution are assumed uncertain and log-normally distributed. The mean and coefficient of variation of the load Gumbel distribution in Table 1 are conditional on the parameters $a_{P}$ (location parameter) and $b_{P}$ (scale parameter):

$$
\begin{aligned}
\mu_{P \mid a_{P}, b_{P}} & =\mu_{a_{P}}+\gamma_{E} \mu_{b_{P}} \\
\delta_{P \mid a_{P}, b_{P}} & =\frac{\pi}{\sqrt{6}} \frac{\mu_{b_{P}}}{\mu_{P \mid a_{P}, b_{P}}},
\end{aligned}
$$


where $\gamma_{E}$ is Euler's constant. It is further assumed that all point loads share the same distribution parameters. Table 1 summarizes the probabilistic input models.

The limit-state function is defined by means of a threshold for the maximum vertical truss deflection, i.e.

$$
g(\boldsymbol{\Theta})=u_{l i m}-u_{\max }(\boldsymbol{\Theta}),
$$

where $u_{\text {lim }}=\{14 \mathrm{~cm}, 16 \mathrm{~cm}, 18 \mathrm{~cm}\}$ are considered, which correspond to system failure probabilities of $\mathbb{P}(F)=\left\{6.2 \cdot 10^{-3}, 1 \cdot 10^{-3}, 1.7 \cdot 10^{-4}\right\}$ (estimated with Monte Carlo, estimator coefficient of variation $\leq 5 \%$ ). In view of the Bayesian interpretation of the variable sets $\boldsymbol{\Theta}_{A}$ (irreducible) and $\boldsymbol{\Theta}_{B}$ (data available,

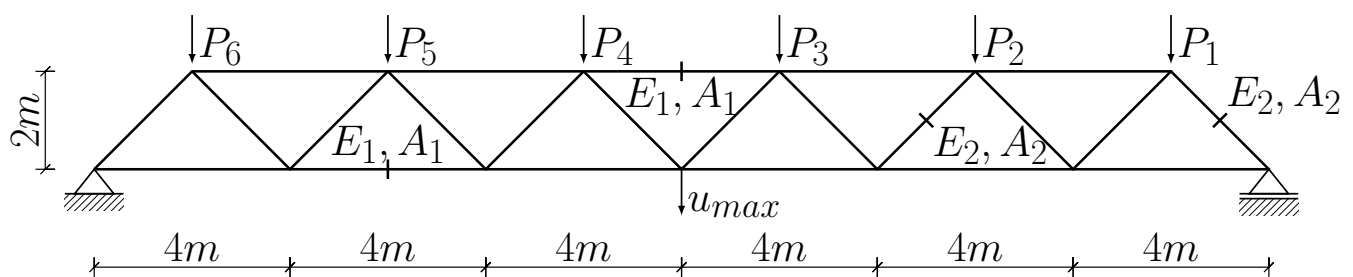

Figure 5: 2-D truss example.

reducible), we choose the latter to comprise quantities which are typically reducible by means of measurements (such as material properties) or archive data (such as load hyperparameters). Thus, we select $\boldsymbol{\Theta}_{B}=\left[E_{1}, A_{1}, E_{2}, A_{2}, a_{P}, b_{P}\right]$ and $\boldsymbol{\Theta}_{A}=\left[P_{1}, \ldots, P_{6}\right]$. For this example, we use the global surrogate mod-

Table 1: Input variable definitions of the elastic truss.

\begin{tabular}{lccc}
\hline Quantity & Distribution & Mean $\mu$ & $\mathrm{CoV} \delta$ \\
\hline$A_{1}\left[m^{2}\right]$ & Log-Normal & $2 \cdot 10^{-3}$ & 0.1 \\
$A_{2}\left[m^{2}\right]$ & Log-Normal & $1 \cdot 10^{-3}$ & 0.1 \\
$E_{1}, E_{2}[\mathrm{~Pa}]$ & Log-Normal & $2.1 \cdot 10^{11}$ & 0.1 \\
$P_{1}-P_{6}[N]$ & Gumbel & $\mu_{P \mid a_{P}, b_{P}}$ & $\delta_{P \mid a_{P}, b_{P}}$ \\
$a_{P}[N]$ & Log-Normal & 46624 & 0.2 \\
$b_{P}[N]$ & Log-Normal & 3375 & 0.2 \\
\hline
\end{tabular}

elling strategy, as the truss behaves only mildly nonlinear. Both level 1- and level 2-experimental designs are obtained via latin hypercube sampling. $n_{1}=200$ samples are used to construct the level 1-LRA, while $n_{2}=1000$ points are used to evaluate the level 2-surrogate. The analysis is repeated 20 times redrawing random level 1-experimental designs which yields the estimator statistics provided in Figures 6 and 7 . These are computed for the difference of the surrogate-based estimator from the direct Monte-Carlo (DMC) reference solution, i.e.

$$
\epsilon_{Q}=Q-Q_{D M C} .
$$

All reliability analyses haven been performed with the first-order reliability method (FORM). For the reference solution, $n_{D M C}=2 \cdot 10^{5}$ samples have been used implying the solution of $8 \cdot 10^{5}$ reliability problems of dimension 6 (since the type $B$-variable space has dimension 6, see Section 2.3). While both PCE and LRA-based approaches capture the variable importance ranking correctly, the LRA-based approach performs slightly but consistently better in the mean compared to the PCE-based approach. The least important variables $E_{2}, A_{2}$ are estimated with the smallest error mean and variance, which, however, is due to their small true magnitude. Intuitively, estimation accuracy and index magnitude should depend on one another reciprocally; the larger the index the more accurate it is estimated based on a given set of samples. Indeed, relative to their respective magnitudes, the indices of the most important indices $a_{P}$ and $b_{P}$ are estimated more accurately. 


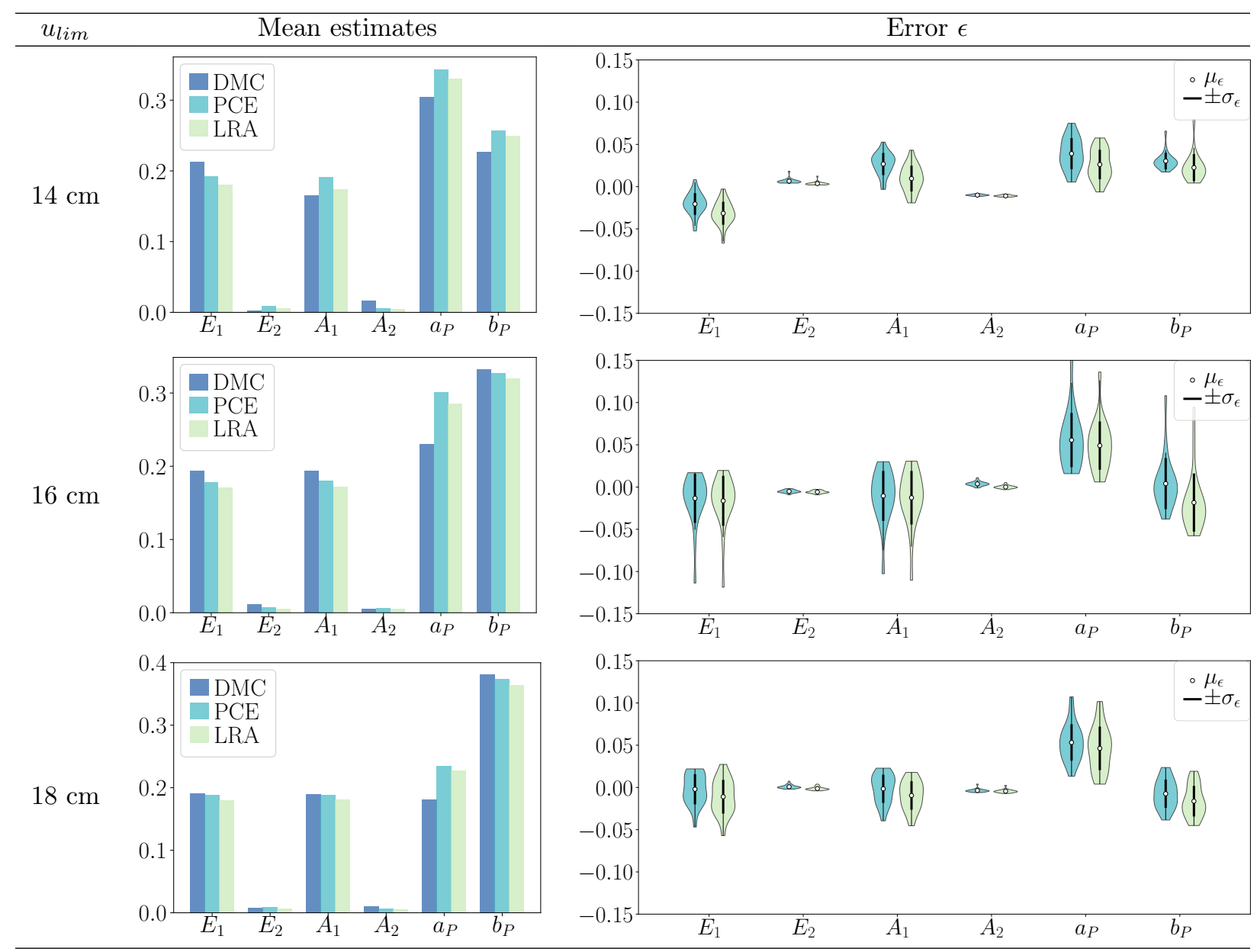

Figure 6: $\log P_{F}$ first-order Sobol' indices: mean estimates and errors $\left(n_{1}=200, n_{2}=1000\right)$. 


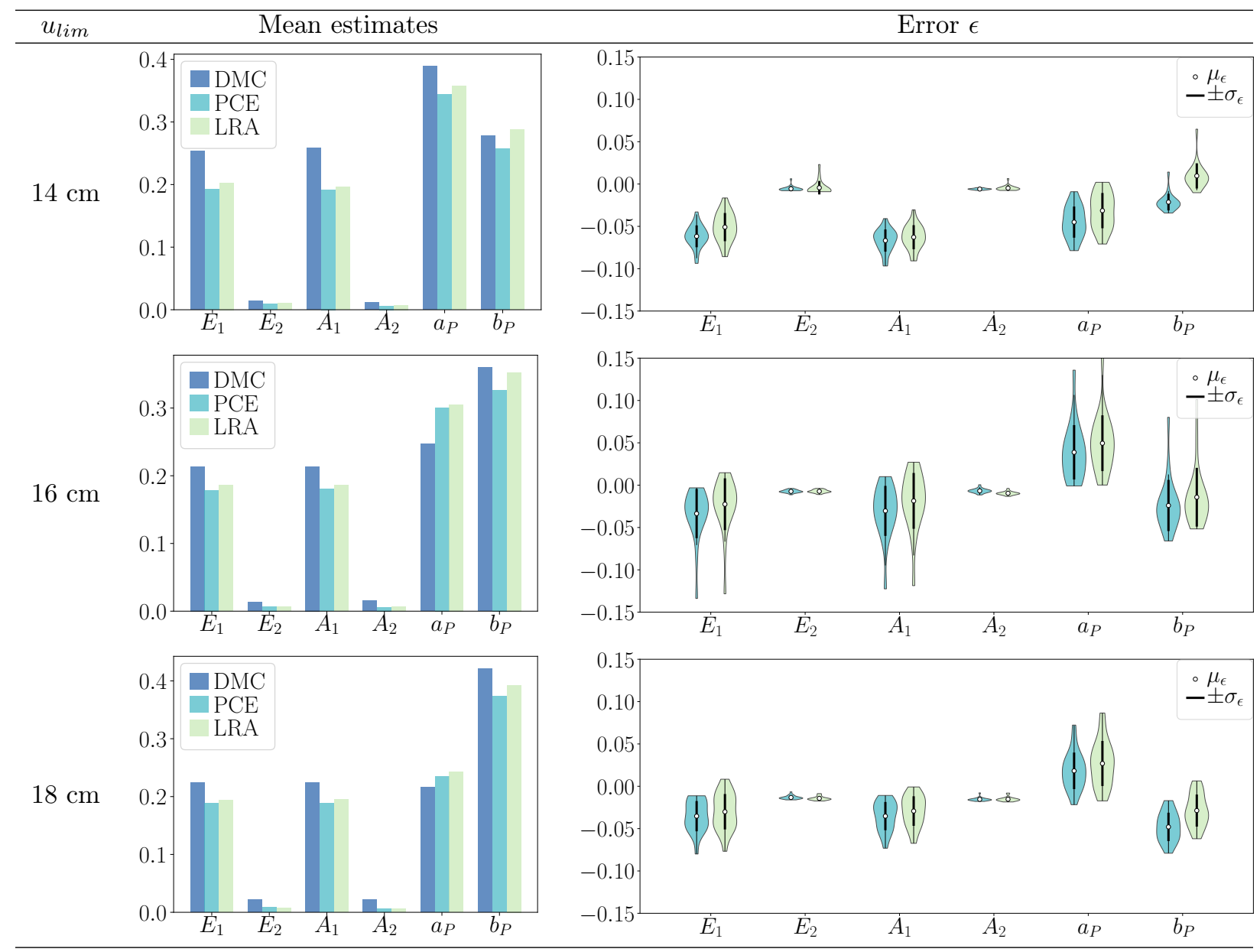

Figure 7: $\log P_{F}$ Total-effect indices: mean estimates and errors $\left(n_{1}=200, n_{2}=1000\right)$. 


\subsection{Monopile Foundation}

In this example, we consider a finite element model for the interaction of a monopile foundation of an offshore wind turbine (Figure 8) with stiff, plastic soil. Deterministic parameters of the monopile are its depth $L=30 \mathrm{~m}$, diameter $D=6 \mathrm{~m}$, wall thickness $t=0.07 \mathrm{~m}$, Poisson ratio $\nu=0.3$ and Young's modulus $E=2.1 \cdot 10^{5} \mathrm{MPa}$. The uncertain inputs comprise the lateral load $H$ as well as the undrained shear strength $s$ of the soil and hyperparameters of both quantities. The engineering model setup follows [48] and the probabilistic model considered there has been modified following [49]. $s$ is considered both uncertain and increasing in mean with soil depth $z$. It is thus modelled by a random field with linear mean drift along the soil depth coordinate $z$. Given an underlying stationary Gaussian random field $\tilde{s}(z, \boldsymbol{\Theta})$

$$
\{\tilde{s}(z): 0 \leq z \leq L\} \sim \mathcal{N}\left(0, \sigma_{\tilde{s}}\right),
$$

the non-stationary random field representing the shear strength of the soil can be expressed as

$$
\begin{aligned}
s(z, \boldsymbol{\Theta}) & =s_{0}+s_{1} \sigma^{\prime}(z) \exp \{\tilde{s}(z, \boldsymbol{\Theta})\} \\
& =s_{0}+s_{1} \gamma z \exp \{\tilde{s}(z, \boldsymbol{\Theta})\},
\end{aligned}
$$

where $\gamma$ is the soil unit weight, $\sigma^{\prime}(z)=\gamma z$ is the effective vertical stress, $s_{0}$ is the undrained shear strength at ground level and $s_{1}$ is the drift parameter governing the mean increase of $s$ with increasing soil depth. $\tilde{s}(z, \boldsymbol{\Theta})$ models the intra-site variability. That is, at a given site with known deterministic $s_{0}$ and $s_{1}$, it describes the inherent variability of the undrained shear strength. In order to describe the inter-site uncertainty in $s$, the parameters $s_{0}$ and $s_{1}$ are modeled probabilistically as well. The stationary $\mathrm{RF} \tilde{s}$ is taken to be correlated with exponential-type

$$
\rho_{\tilde{s} \tilde{s}}\left(z^{\prime}, z^{\prime \prime}\right)=\exp \left\{-\frac{2\left|z^{\prime}-z^{\prime \prime}\right|}{\theta_{\tilde{s}}}\right\},
$$

with vertical soil scale of fluctuation $\theta_{\tilde{s}}=1.9 \mathrm{~m}[50]$ and standard deviation $\sigma_{\tilde{s}}=0.3[50,51]$. We assume the soil to be stiff and plastic according to the classification provided in [52]. There, the specific soil weight range is given with $17-19 \mathrm{kN} / \mathrm{m}^{3}$, whence we set $\gamma=18 \mathrm{kN} / \mathrm{m}^{3}$. The mean cohesion range is given with $20-50 \mathrm{kN} / \mathrm{m}^{2}$ by [52] while [53] lists the mean range of the undrained shear strength ratio $s_{u} / \sigma^{\prime}$ as $0.23-1.4$. We fit log-normal distributions for $s_{0}$ and $s_{1}$ by setting the $10 \%$ and $90 \%$ quantiles of the distributions equal to the lower and upper bounds of these ranges. The resulting parameters are detailed in Table 2 along with uncertain parameters for the load $H$, namely $\mu_{H}$ and $\sigma_{H}$. The mean and coefficient of variation of the load Gumbel distribution in Table 2 are conditional on the parameters $a_{H}$ (location parameter) and $b_{H}$ (scale parameter) according to Eqs. (29) and (30). $\tilde{s}$ is simulated by means of the midpoint method. That

Table 2: Input variable definitions of the monopile foundation.

\begin{tabular}{lccc}
\hline Input & Distribution & Mean $\mu$ & $\mathrm{CoV} \delta$ \\
\hline $\boldsymbol{\xi}[-]$ & Standard-Normal & $\mathbf{0}$ & n.d. $\left(\boldsymbol{\Sigma}_{\boldsymbol{\xi} \boldsymbol{\xi}}=\boldsymbol{I}_{n \times n}\right)$ \\
$s_{0}[k P a]$ & Log-Normal & 33.7094 & 0.3692 \\
$s_{1}[k P a]$ & Log-Normal & 0.7274 & 0.8019 \\
$H[k N]$ & Gumbel & $\mu_{P \mid a_{H}, b_{H}}$ & $\delta_{P \mid a_{H}, b_{H}}$ \\
$a_{H}[k N]$ & Log-Normal & 2274.97 & 0.2 \\
$b_{H}[k N]$ & Log-Normal & 225.02 & 0.2 \\
\hline
\end{tabular}

is, the spatial domain $[0, L]$ is discretized with $n$ spatial elements and $\tilde{s}$ is represented by means of $n$ random variables with joint distribution $\mathcal{N}\left(\mathbf{0}, \boldsymbol{\Sigma}_{\tilde{s} \tilde{s}}\right)$. The random variables represent the random field values at the element midpoints. Thus, the covariance matrix $\boldsymbol{\Sigma}_{\tilde{s} \tilde{s}}$ is computed by evaluating $\sigma_{\tilde{s}}^{2} \rho_{\tilde{s} \tilde{s}}\left(z^{\prime}, z^{\prime \prime}\right)$ at the element midpoints. The number of elements is chosen such that $95 \%$ of the inherent $\mathrm{RF}$ variability is captured by the $\mathrm{RF}$ discretization, leading to $n=82$ in this example. Therefore, the total input dimension is $d=87$. As the 


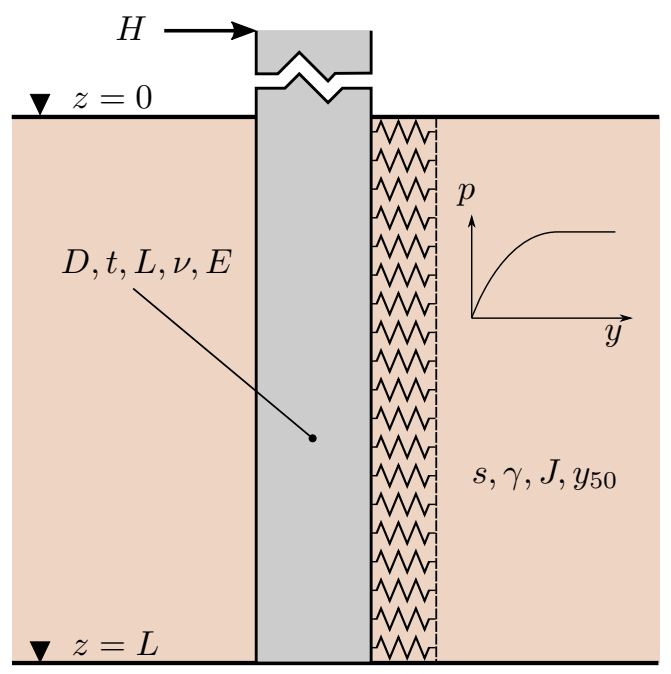

Figure 8: Wind turbine monopile foundation [48].

surrogate modeling approach requires independent inputs, the midpoint random variables are transformed to independent standard normal random variables, denoted as $\boldsymbol{\xi}$, by means of the Nataf transform. The model output $Y=\mathcal{Y}(\boldsymbol{\Theta})$ is the maximum occurring stress in the foundation. The limit-state function is given by

$$
g(\mathbf{\Theta})=\sigma_{c r i t}-\mathcal{Y}(\boldsymbol{\Theta}),
$$

where three different stress thresholds $\sigma_{\text {crit }}=\{80 \mathrm{MPa}, 100 \mathrm{MPa}, 120 \mathrm{MPa}\}$ are considered with corresponding system failure probabilities $\mathbb{P}(F)=\left\{3.0 \cdot 10^{-3}, 3.6 \cdot 10^{-4}, 8.0 \cdot 10^{-5}\right\}$ (estimated with MC and $\mathrm{CoV}=\{0.0409,0.1187,0.2500\})$. For this example, we consider a scenario in which uncertainties about all the input hyperparameters as well as the inherent variability of the shear strength can be reduced through additional data, i.e. $\boldsymbol{\Theta}_{B}=\left[\boldsymbol{\xi}, a_{H}, b_{H}, s_{0}, s_{1}\right]$. This leaves the inherent load variability as the only remaining aleatory input, i.e. $\boldsymbol{\Theta}_{A}=H$. This example has proven extremely challenging for common global surrogate models (polynomial basis surrogates, adaptive kriging surrogates). Therefore, the local surrogate modelling strategy is chosen, which, in this case, is an efficient choice since the limit-state function to be approximated at each $\boldsymbol{\Theta}_{B}$-sample is one-dimensional. As little as four training points are necessary per $\boldsymbol{\Theta}_{B}$-sample. Based on the local surrogate, the corresponding one-dimensional reliability problem can be solved using a bisectionor Newton-procedure due to the monotonicity of the limit-state-function the same SRM is applied for the reference solution with the original model $\mathcal{Y}$ ). Accurate estimates of the conditional reliability sensitivities are achieved with an overall 2000 evaluations of the limit-state-function for the second example with the introduced framework. This is a conservative choice and satisfying accuracy may be achieved with a considerably lower number of model evaluations as is evident from Figures $11 \& 12$.

The algorithm is run 20 times redrawing level 2-experimental designs to compute errors $\epsilon$ arising from exploring $\Omega_{\Theta_{B}}$ randomly. These errors are measured against a DMC-based reference solution according to Eq. (31). The reference solution is based on $n_{s}=20000$ independent samples which yields a total of $1.64 \cdot 10^{6}$ reliability problems since $d_{B}=86$. A single reference solution (fixed $\sigma_{\text {crit }}$ ) obtained with a Matlab implementation took approximately 48 hours to complete on 10 Intel Xeon E5-2697 v3 14-core nodes, which emphasizes the need for surrogate modelling-based estimators. In order to assess the random field influence, the sum of all first-order indices of the elements of $\boldsymbol{\xi}$ are reported for both the reference solution and the surrogate-based approach. This is motivated by the impossibility of efficiently estimating a sensitivity index of order 86 with the MC-methods of $[17,18]$. However, the surrogate-based estimators indicate negligible 


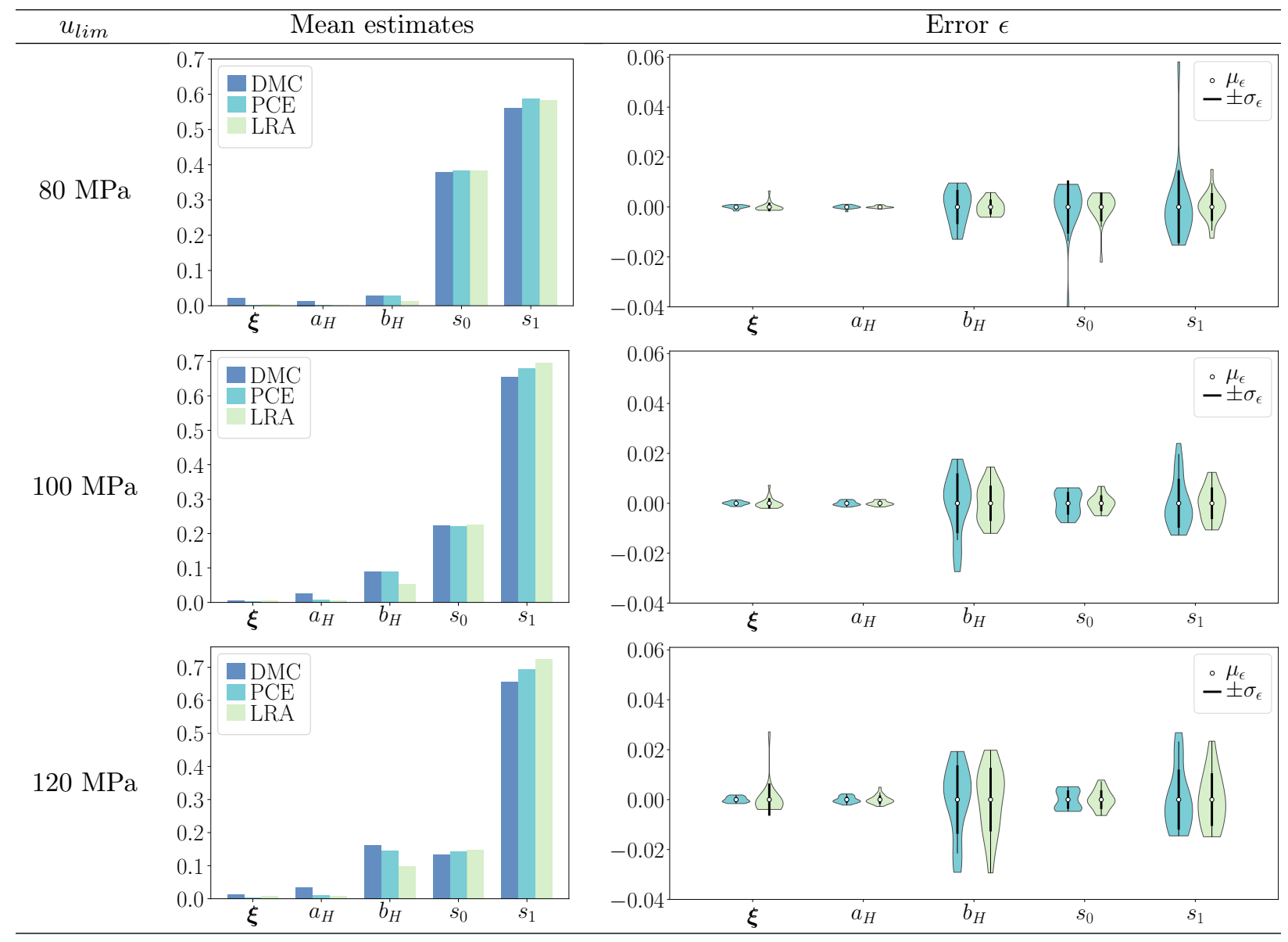

Figure 9: $\log P_{F}$ first-order Sobol' indices: mean estimates and errors $\left(n_{1}=4\right.$ (local), $\left.n_{2}=2000\right)$.

interaction of the elements of $\boldsymbol{\xi}$ (all Sobol' and total-effect indices are virtually identical) thus justifying this approximation.

The sensitivities depicted in Figure 9 and 10 summarize the effect of the inherent random field variability in the order- $n$-indices of the random vector $\boldsymbol{\xi}$. The PCE and LRA level 2 -surrogates yield similar estimates for the reliability sensitivities (Figures 9 and 10): The mean soil shear strength gradient $s_{1}$ is identified as the most influential input to the monopile reliability analysis across all investigated scenarios. The dominance of $s_{1}$ becomes more pronounced at higher critical stress levels. PCE-based sensitivity estimate means are in slightly better agreement with the reference solution than LRA-based estimates. Moreover, PCE-based estimates exhibit consistently smaller variability resulting from the random level 2-experimental design. Error means and variabilities are of comparable magnitude for all computed indices in this example. However, as discussed for the truss example, indices of larger magnitude should be estimated more accurately at a given amount of information. This is true when considering the estimation error relative to the index magnitudes. Then, estimates of the most influential variables $s_{0}$ and $s_{1}$ exhibit comparably small variability due to the random level 2-design of experiments while the less important variables' estimators prove more sensitive in this respect. In Figures $11 \& 12$, the evolution of the error means and variances at different level 2-experimental design sizes $n_{2}$ are depicted. The error converges in mean and standard deviation as $n_{2}$ increases. The error mean apparently is bounded from below which is likely due to a bias in the reference solution which arises as the MC-estimates are not fully converged at $n_{c}=20000$ independent samples. Ultimately, if a decision-maker were to choose whether to acquire data on either of the uncertain model inputs 


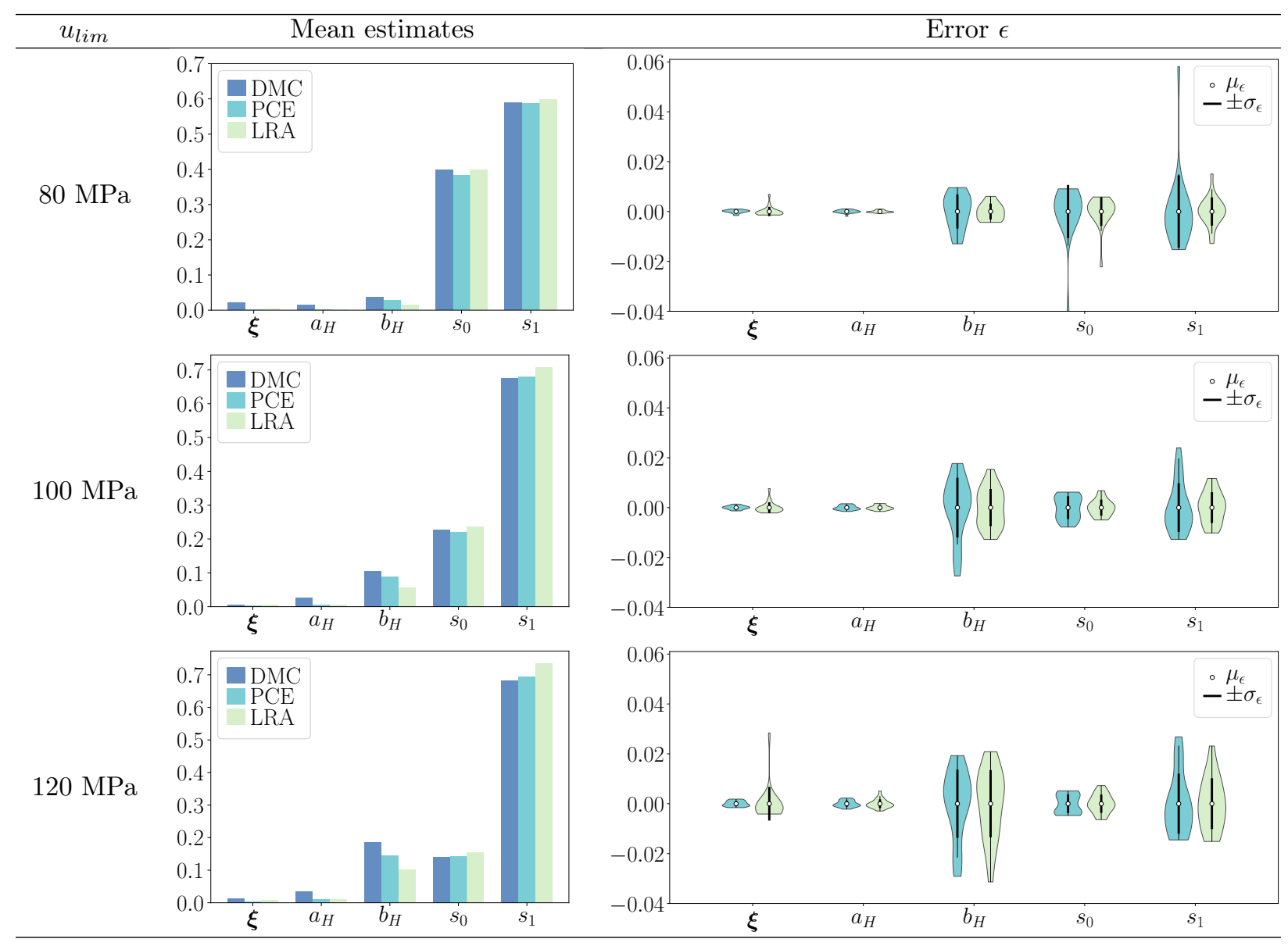

Figure 10: $\log P_{F}$ Total-effect Sobol' indices: mean estimates and errors $\left(n_{1}=4\right.$ (local), $\left.n_{2}=2000\right)$.

and on which input in particular, they should choose $s_{1}$ according to the introduced reliability sensitivity framework. 

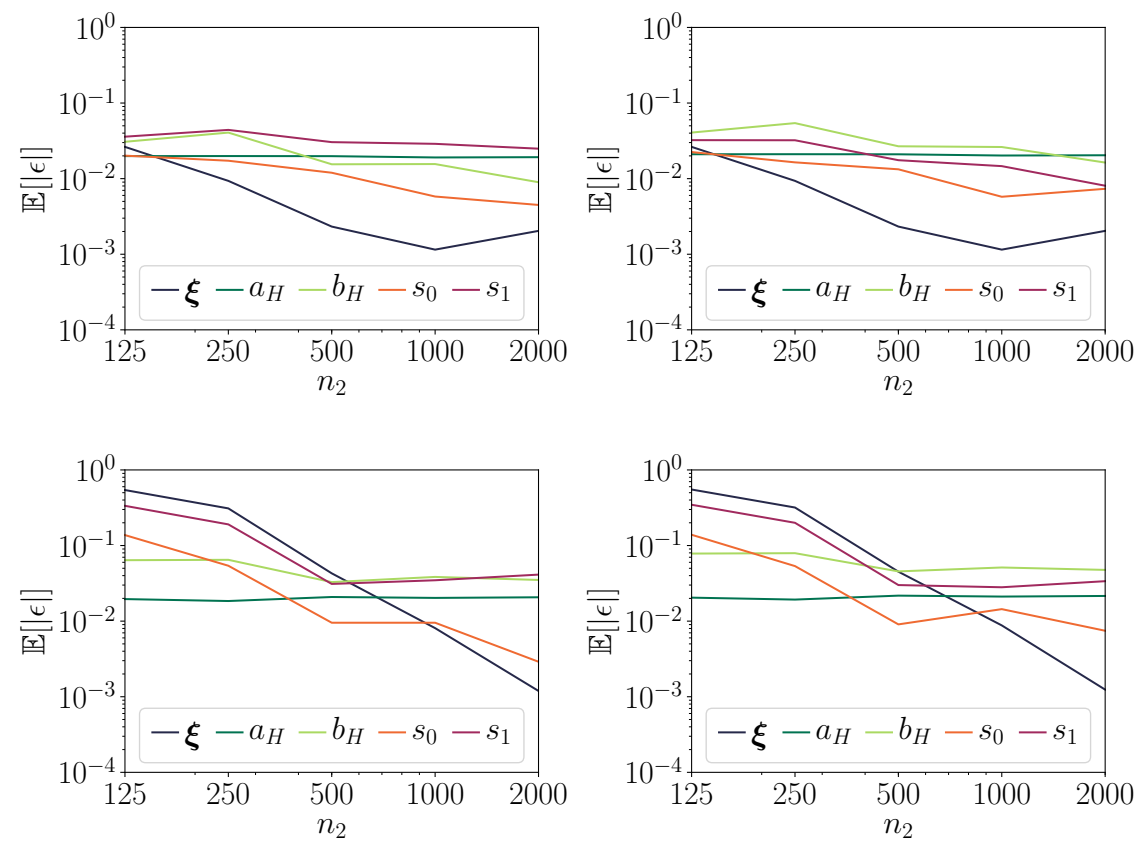

Figure 11: Sobol' (left column) and total-effect indices (right column) error mean vs. level 2 experimental design size $n_{2}$ for PCE- (upper row) and LRA-based (lower row) at $\sigma_{\text {crit }}=100 \mathrm{MPa}$.
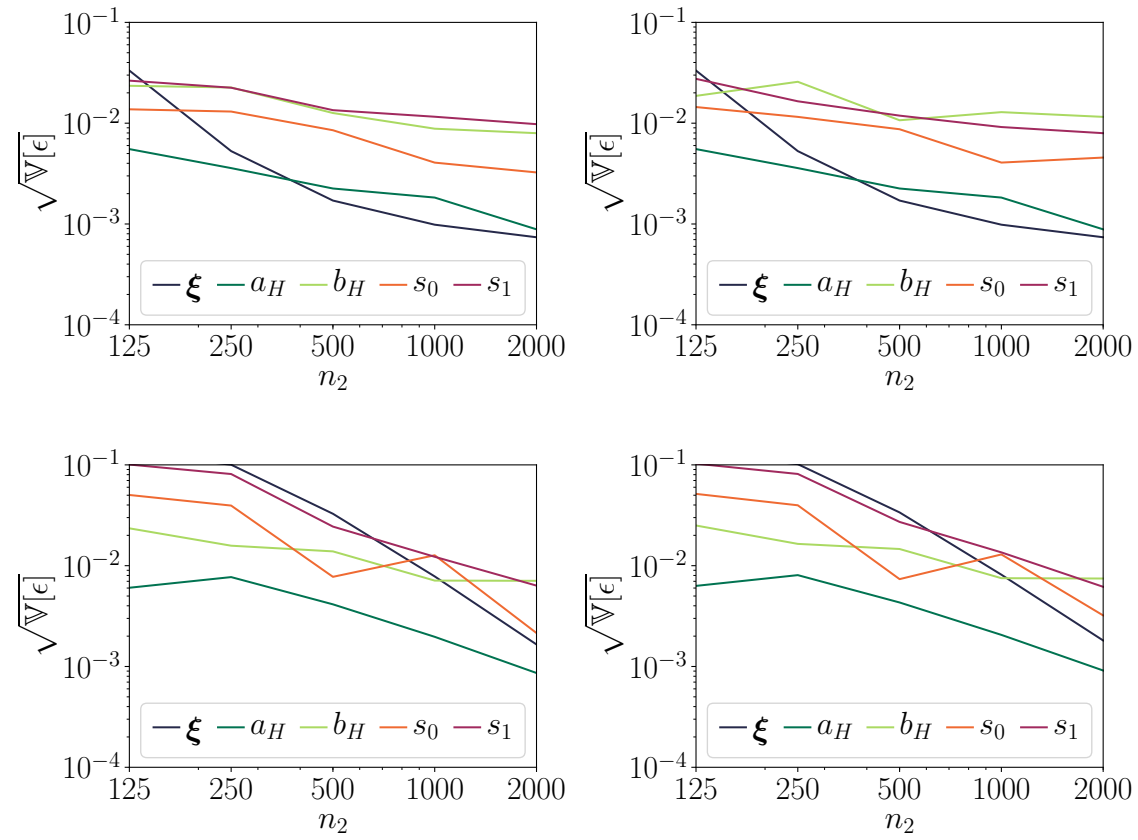

Figure 12: Sobol' (left column) and total-effect indices (right column) error standard deviation vs. level 2 experimental design size $n_{2}$ for PCE- (upper row) and LRA-based (lower row) at $\sigma_{\text {crit }}=100 \mathrm{MPa}$. 


\section{Concluding Remarks}

In this paper we describe a framework for reliability sensitivity analysis whose core is a novel, variancebased sensitivity index tailored to reliability analysis in the presence of multi-uncertainty. Multi-uncertainty refers to a separation of uncertain model inputs in different categories, e.g. aleatory and epistemic. The interest then lies in expressing the sensitivity of the probability of failure-estimate to the epistemic inputs. We devise a flexible two-level surrogate modelling approach which allows for a cost-efficient estimation of the proposed index. The approach relies on either building a global surrogate model over the entirety of input variables once and for all (global strategy) or on repeatedly recomputing cheaper, lower-dimensional surrogates (local strategy). An analysis of computational cost for both local and global surrogate modelling strategy has been carried out in dependence on how the model input is divided in reducible and irreducible.

We demonstrate the novel approach by means of two examples. In the elastic truss example, the sensitivity indices were estimated using a global low-rank approximation in level 1 at a total cost of 200 original model evaluations. The LRAs are more suitable for global surrogate-driven reliability computations (level 1) compared to the PCEs, as discussed in [40]. For the geotechnical example, namely a monopile foundation, a local surrogate modelling approach based on PCEs has been adopted in level 1 yielding a total cost of 8000 original model evaluations. In level 2, the sparse PCEs perform slightly better than the LRA. Sensitivity estimates for both examples were validated with sampling-based reference estimates showing that the important input's indices are estimated accurately by the method. While in this paper only two possible choices for the level 1 surrogate are discussed, virtually any surrogate modelling technique could be applied here.

\subsection{Discussion}

The contribution of the proposed sensitivity index is threefold. First, it focusses the sensitivity analysis on a variable subset that is of interest in the presence of multi-uncertainty and data assimilation applications. Second, it represents a direct sensitivity measure for the probability of failure magnitude as opposed to indices based on the indicator function that rank influence on the failure hypersurface shape. Finally, the new index facilitates the entirely surrogate-driven computation of reliability sensitivities by smoothening the indicator function discontinuity through an integral formulation.

In accord with intuition, estimators of sensitivity indices with small magnitude exhibit smaller errors comapred to those of large magnitude. Relative to the magnitude, the opposite is true: At fixed amount of information (here: the level 1-training set size) the sensitivity indices with larger magnitude are estimated more reliably. Moreover, the variable importance ranking is accurately captured in all numerical examples. The problem dimension, which can be handled by the approach is guided by the surrogate modelling techniques chosen in both levels. Using an arbitrary surrogate model that is capable of addressing highdimensional problems in level 1 and LRAs or the recently introduced PLS-driven PCEs [54] in level 2, it is applicable up to several thousand input variables.

At a fixed accuracy (with respect to $\mathcal{Y}$-model output), the cost of the global surrogate modelling strategy remains constant irrespective of the fraction of reducible and irreducible uncertainty in the model input. The same is not true for the local strategy where two counteracting effects lead to the local strategy being most efficient if either of the uncertainty types dominates the model input. The local modelling strategy is more flexible and expected to perform well even for strongly nonlinear models, when the global modelling strategy may deteriorate. In such case, the required number of samples for a sufficiently accurate global surrogate model may overcompensate the strategy-based savings the global modelling strategy offers and render the local strategy the better choice. Therefore, the model under consideration should govern the decision which of either approach is used. Generally, we recommend to apply the local surrogate modelling strategy when the model under consideration exhibits significant nonlinear behaviour.

\subsection{Outlook}

The experimental design for the level 1-surrogate dominates the computational cost of the approach. The accuracy of the overall method is controlled by the capability of the level 1-surrogate to capture the 
tail behaviour of the model response and that of the level 2-surrogate to capture the second moment of its derived response (the conditional probability of failure). Thus, the level 1-surrogate represents the most crucial element of the framework both with respect to accuracy and computational cost. Next to the choice of surrogate, the level 1-performance would likely benefit from a more guided selection of experimental design points in level 1, which addresses the method's requirement of surrogates to remain accurate close to $g=0$ rather than minimize global error measures.

Moreover, the introduced sensitivity measure may be connected to decision-oriented sensitivity analysis and in particular the concept of expected value of partial perfect information (EVPPI). It is known that the classical first-order variance-based sensitivity measure coincides with the EVPPI for a special decision context (with a quadratic loss function) [55]. Based on this, the proposed framework may be adapted to facilitate computation of more relevant loss functions associated within decision analysis, which in turn promotes its applicability for decision support.

\section{Acknowledgments}

This work was supported by the Deutsche Forschungsgemeinschaft (DFG) in the framework of the priority programme SPP 1886 by grant STR 1140/6-1.

\section{Appendix A. Connecting the proposed Sobol' index to its indicator function-based counter- part}

Here, we discuss the connection of the novel sensitivity indices to the the measures proposed by [20]. In particular, we show that the Sobol' index of the condititonal probability of failure (without logarithmic transformation) is identical to the Sobol' index of the indicator function $\mathrm{I}(g \leq 0)$ up to a normalizing constant. In this case, the quantity of interest reads

$$
Q=P_{F}\left(\boldsymbol{\theta}_{B}\right)=\mathbb{E}_{\boldsymbol{\Theta}_{A}}\left[\mathrm{I}\left(g\left(\boldsymbol{\Theta}_{A}, \boldsymbol{\Theta}_{B}\right) \leq 0\right) \mid \boldsymbol{\Theta}_{B}=\boldsymbol{\theta}_{B}\right],
$$

while in [20], $Q=\mathrm{I}(g(\boldsymbol{\Theta}) \leq 0)$ is used. Consider now an arbitrary subset of $\boldsymbol{\Theta}_{B}$ which is denoted by $\boldsymbol{\Theta}_{\mathcal{B}}$ and its complement $\boldsymbol{\Theta}_{\sim \mathcal{B}}$ such that $\boldsymbol{\Theta}_{B}=\left\{\boldsymbol{\Theta}_{\mathcal{B}}, \boldsymbol{\Theta}_{\sim \mathcal{B}}\right\}$. The Sobol' index of $Q=P_{F}$ for the variable subset $\boldsymbol{\Theta}_{\mathcal{B}}$ is given by

$$
S_{P_{F}, \mathcal{B}}=\frac{\mathbb{V}_{\boldsymbol{\Theta}_{\mathcal{B}}}\left[\mathbb{E}_{\boldsymbol{\Theta}_{\sim \mathcal{B}}}\left[\mathbb{E}_{\boldsymbol{\Theta}_{A}}\left[\mathrm{I}(g \leq 0) \mid \boldsymbol{\Theta}_{B}\right] \mid \boldsymbol{\Theta}_{\mathcal{B}}\right]\right]}{\mathbb{V}_{\boldsymbol{\Theta}_{B}}\left[\mathbb{E}_{\boldsymbol{\Theta}_{A}}\left[\mathrm{I}(g \leq 0) \mid \boldsymbol{\Theta}_{B}\right]\right]},
$$

while the Sobol' for $Q=\mathrm{I}(g(\boldsymbol{\Theta}) \leq 0)$ reads

$$
S_{\mathrm{I}(g \leq 0), \mathcal{B}}=\frac{\mathbb{V}_{\boldsymbol{\Theta}_{\mathcal{B}}}\left[\mathbb{E}_{\boldsymbol{\Theta}_{A, \sim \mathcal{B}}}\left[\mathrm{I}(g \leq 0) \mid \boldsymbol{\Theta}_{\mathcal{B}}\right]\right]}{\mathbb{V}_{\boldsymbol{\Theta}}[\mathrm{I}(g \leq 0)]} .
$$

Here, $\boldsymbol{\Theta}_{A, \sim \mathcal{B}}$ denotes the union of $\boldsymbol{\Theta}_{A}$ and $\boldsymbol{\Theta}_{\sim \mathcal{B}}$. Then, we have

$$
\begin{aligned}
& S_{P_{F}, \mathcal{B}}=\frac{1}{\mathbb{V}\left[P_{F}\right]} \mathbb{V}_{\boldsymbol{\Theta}_{\mathcal{B}}}\left[\int_{\Omega_{\boldsymbol{\Theta}_{\sim \mathcal{B}}}} \pi\left(\boldsymbol{\theta}_{\sim \mathcal{B}} \mid \boldsymbol{\Theta}_{\mathcal{B}}\right) \int_{\Omega_{\boldsymbol{\Theta}_{A}}} \mathrm{I}\left(g\left(\boldsymbol{\theta}_{A}, \boldsymbol{\theta}_{\sim \mathcal{B}}, \boldsymbol{\Theta}_{\mathcal{B}}\right) \leq 0\right) \pi\left(\boldsymbol{\theta}_{A} \mid \boldsymbol{\theta}_{\sim \mathcal{B}}, \boldsymbol{\Theta}_{\mathcal{B}}\right) \mathrm{d} \boldsymbol{\theta}_{A} \mathrm{~d} \boldsymbol{\theta}_{\sim \mathcal{B}}\right] \\
& =\frac{1}{\mathbb{V}\left[P_{F}\right]} \mathbb{V}_{\boldsymbol{\Theta}_{\mathcal{B}}}\left[\int_{\Omega_{\boldsymbol{\Theta}_{A, \sim \mathcal{B}}}} \mathrm{I}\left(g\left(\boldsymbol{\theta}_{A, \sim \mathcal{B}}, \boldsymbol{\Theta}_{\mathcal{B}}\right) \leq 0\right) \pi\left(\boldsymbol{\theta}_{A, \sim \mathcal{B}} \mid \boldsymbol{\Theta}_{\mathcal{B}}\right) \mathrm{d} \boldsymbol{\theta}_{A, \sim \mathcal{B}}\right] \\
& =\frac{\mathbb{V}[I(g \leq 0)]}{\mathbb{V}\left[P_{F}\right]} \frac{\mathbb{V}_{\boldsymbol{\Theta}_{\mathcal{B}}}\left[\mathbb{E}_{\boldsymbol{\Theta}_{A, \sim \mathcal{B}}}\left[\mathrm{I}(g \leq 0) \mid \boldsymbol{\Theta}_{\mathcal{B}}\right]\right]}{\mathbb{V}_{\boldsymbol{\Theta}}[\mathrm{I}(g \leq 0)]} \\
& =\frac{\mathbb{V}[I(g \leq 0)]}{\mathbb{V}\left[P_{F}\right]} S_{\mathrm{I}(g \leq 0), \mathcal{B}} .
\end{aligned}
$$


The rescaling constant connecting the multi-uncertainty Sobol' index to its indicator function-based counterpart is the ratio of variance fractions contributed by all the input variables and the type B-variables only. This result is somewhat intuitive as the multi-uncertainty Sobol' index is defined with respect to the variance contributed by the type B-variables only.

\section{References}

[1] O. Ditlevsen, H. O. Madsen, Structural reliability methods, John Wiley \& Sons Ltd, 1996.

[2] A. Der Kiureghian, First-and second-order reliability methods, in: E. Nikolaidis, D. M. Ghiocel, S. Singhal (Eds.), Engineering Design Reliability Handbook, CRC Press, Boca Raton, FL, 2005.

[3] C. G. Bucher, Adaptive sampling — an iterative fast monte carlo procedure, Structural Safety 5 (1988) 119 - 126.

[4] V. Dubourg, B. Sudret, F. Deheeger, Metamodel-based importance sampling for structural reliability analysis, Probabilistic Engineering Mechanics 33 (2013) 47 - 57.

[5] P. Koutsourelakis, H. Pradlwarter, G. Schuëller, Reliability of structures in high dimensions, part i: algorithms and applications, Probabilistic Engineering Mechanics 19 (2004) 409 - 417.

[6] S.-K. Au, J. L. Beck, Estimation of small failure probabilities in high dimensions by subset simulation, Probabilistic engineering mechanics 16 (2001) 263-277.

[7] I. Papaioannou, C. Papadimitriou, D. Straub, Sequential importance sampling for structural reliability analysis, Structural Safety $62(2016) 66-75$.

[8] P. Beaurepaire, H. Jensen, G. Schuëller, M. Valdebenito, Reliability-based optimization using bridge importance sampling, Probabilistic Engineering Mechanics 34 (2013) 48-57.

[9] B. Echard, N. Gayton, M. Lemaire, AK-MCS: An active learning reliability method combining kriging and Monte Carlo simulation, Structural Safety 33 (2011) $145-154$.

[10] B. Sudret, Meta-models for structural reliability and uncertainty quantification, in: K. K. Phoon, M. Beer, S. T. Quek, S. D. Pang (Eds.), Proc. Fifth Asian-Pacific Symposium on Structural Reliability and its Applications (5APSSRA), Singapore, 2012.

[11] A. Der Kiureghian, O. Ditlevsen, Aleatory or epistemic? Does it matter?, Structural Safety 31 (2009) 105-112.

[12] B. Adams, Bauman, L.E., W. Bohnhoff, K. Dalbey, M. Ebeida, J. Eddy, M. Eldred, P. Hough, K. Hu, J. Jakeman, J. Stephens, L. Swiler, D. Vigil, , T. Wildey, Dakota, A Multilevel Parallel Object-Oriented Framework for Design Optimization, Parameter Estimation, Uncertainty Quantification, and Sensitivity Analysis: User's Manual, SAND20144633, Sandia National Laboratories, November 2015.

[13] V. Chabridon, M. Balesdent, J.-M. Bourinet, J. Morio, N. Gayton, Reliability-based sensitivity estimators of rare event probability in the presence of distribution parameter uncertainty, Reliability Engineering \& System Safety (2018).

[14] I. Papaioannou, K. Breitung, D. Straub, Reliability sensitivity analysis with Monte Carlo methods, CRC Press, pp. $5335-5342$.

[15] I. Sobol', Sensitivity estimates for nonlinear mathematical models, Math. Modeling \& Comp. Exp 1 (1993) $407-414$.

[16] T. Homma, A. Saltelli, Importance measures in global sensitivity analysis of nonlinear models, Reliability Engineering \& System Safety 52 (1996) $1-17$

[17] M. J. Jansen, Analysis of variance designs for model output, Computer Physics Communications 117 (1999) 35 - 43.

[18] A. Saltelli, P. Annoni, I. Azzini, F. Campolongo, M. Ratto, S. Tarantola, Variance based sensitivity analysis of model output. design and estimator for the total sensitivity index, Computer Physics Communications 181 (2010) 259-270.

[19] E. Borgonovo, A new uncertainty importance measure, Reliability Engineering \& System Safety 92 (2007) 771 - 784.

[20] L. Li, Z. Lu, J. Feng, B. Wang, Moment-independent importance measure of basic variable and its state dependent parameter solution, Structural Safety 38 (2012) $40-47$.

[21] I. Papaioannou, K. Breitung, D. Straub, Reliability sensitivity estimation with sequential importance sampling, Structural Safety 75 (2018) $24-34$.

[22] B. Iooss, P. Lemaître, A review on global sensitivity analysis methods, in: C. Meloni, G. Dellino (Eds.), Uncertainty management in Simulation-Optimization of Complex Systems: Algorithms and Applications, Springer, 2015.

[23] A. Saltelli, K. Chan, E. Scott, Sensitivity Analysis, John Wiley \& Sons, Inc., 2000.

[24] Y.-T. Wu, Computational methods for efficient structural reliability and reliability sensitivity analysis, AIAA journal 32 (1994) 1717-1723.

[25] S. Song, Z. Lu, H. Qiao, Subset simulation for structural reliability sensitivity analysis, Reliability Engineering \& System Safety 94 (2009) $658-665$.

[26] V. Dubourg, B. Sudret, Meta-model-based importance sampling for reliability sensitivity analysis, Structural Safety 49 (2014) 27 - 36. Special Issue In Honor of Professor Wilson H. Tang.

[27] S. Au, Reliability-based design sensitivity by efficient simulation, Computers \& structures 83 (2005) $1048-1061$.

[28] M.Hohenbichler, R.Rackwitz, Sensitivity and importance measures in structural reliability, Civil Engineering Systems 3 (1986) 203-209.

[29] T. Kim, J. Song, Generalized reliability importance measure (GRIM) using gaussian mixture, Reliability Engineering \& System Safety 173 (2018) 105-115.

[30] P. Wang, Z. Lu, Z. Tang, An application of the kriging method in global sensitivity analysis with parameter uncertainty, Applied Mathematical Modelling 37 (2013) 6543 - 6555.

[31] J. Morio, Influence of input PDF parameters of a model on a failure probability estimation, Simulation Modelling Practice and Theory 19 (2011) $2244-2255$. 
[32] V. Maume-Deschamps, I. Niang, Estimation of quantile oriented sensitivity indices, Statistics \& Probability Letters 134 (2018) $122-127$.

[33] P. Lemaitre, E. Sergienko, A. Arnaud, N. Bousquet, F. Gamboa, B. Iooss, Density modification-based reliability sensitivity analysis, Journal of Statistical Computation and Simulation 85 (2015) 1200-1223.

[34] D. Xiu, G. E. Karniadakis, The Wiener-Askey polynomial chaos for stochastic differential equations, SIAM Journal on Scientific Computing 24 (2002) 619-644.

[35] M. Berveiller, B. Sudret, M. Lemaire, Stochastic finite element: a non intrusive approach by regression, European Journal of Computational Mechanics 15 (2006) 81-92.

[36] G. Blatman, B. Sudret, Adaptive sparse polynomial chaos expansion based on least-angle regression, Journal of Computational Physics 230 (2011) $2345-2367$.

[37] L. Grasedyck, D. Kressner, C. Tobler, A literature survey of low-rank tensor approximation techniques, GAMMMitteilungen 36 (2013) 53-78.

[38] F. L. Hitchcock, The expression of a tensor or a polyadic as a sum of products, Journal of Mathematics and Physics 6 (1927) 164-189.

[39] M. Chevreuil, R. Lebrun, A. Nouy, P. Rai, A least-squares method for sparse low rank approximation of multivariate functions, SIAM/ASA Journal on Uncertainty Quantification 3 (2015) 897-921.

[40] K. Konakli, B. Sudret, Polynomial meta-models with canonical low-rank approximations: Numerical insights and comparison to sparse polynomial chaos expansions, Journal of Computational Physics 321 (2016) 1144-1169.

[41] B. Sudret, Global sensitivity analysis using polynomial chaos expansions, Reliability Engineering \& System Safety 93 (2008) $964-979$

[42] K. Konakli, B. Sudret, Global sensitivity analysis using low-rank tensor approximations, Reliability Engineering \& System Safety 156 (2016) $64-83$.

[43] S. Marelli, B. Sudret, An active-learning algorithm that combines sparse polynomial chaos expansions and bootstrap for structural reliability analysis, Structural Safety 75 (2018) $67-74$.

[44] I. Papaioannou, M. Ehre, D. Straub, Efficient PCE representations for reliability analysis in high dimensions, in: J. Song (Ed.), Proceedings of the 19th working conference of the IFIP Working Group 7.5 on Reliability and Optimization of Structural Systems, ETH Zürich, 2018.

[45] R. Schöbi, B. Sudret, Global sensitivity analysis in the context of imprecise probabilities (p-boxes) using sparse polynomial chaos expansions, Reliability Engineering \& System Safety (2018).

[46] S. H. Lee, B. M. Kwak, Response surface augmented moment method for efficient reliability analysis, Structural Safety 28 (2006) $261-272$.

[47] M. Ehre, iason Papaioannou, D. Straub, Efficient estimation of variance-based reliability sensitivites in the presence of multi-uncertainty, in: J. Song (Ed.), Proceedings of the 19th working conference of the IFIP Working Group 7.5 on Reliability and Optimization of Structural Systems, ETH Zürich, 2018.

[48] I. Depina, I. Papaioannou, D. Straub, G. Eiksund, Coupling the cross-entropy with the line sampling method for risk-based design optimization, Structural and Multidisciplinary Optimization 55 (2017) 1589-1612.

[49] S.-H. Jiang, I. Papaioannou, D. Straub, Bayesian updating of slope reliability in spatially variable soils with in-situ measurements, Engineering Geology 239 (2018) 310 - 320.

[50] K.-K. Phoon, F. H. Kulhawy, Characterization of geotechnical variability, Canadian Geotechnical Journal 36 (1999) $612-624$.

[51] K.-K. Phoon, F. H. Kulhawy, Evaluation of geotechnical property variability, Canadian Geotechnical Journal 36 (1999) $625-639$.

[52] R. Rackwitz, Reviewing probabilistic soils modelling, Computers and Geotechnics 26 (2000) 199 - 223.

[53] Z. Cao, Y. Wang, D. Li, Quantification of prior knowledge in geotechnical site characterization, Engineering Geology 203 (2016) $107-116$

[54] I. Papaioannou, M. Ehre, D. Straub, PLS-based adaptation for efficient PCE representation in high dimensions, www.era.bgu.tum.de/fileadmin/w00bkd/www/Papers/2018_PLS_PCE_manuscript.pdf, 2018. Under review.

[55] J. E. Oakley, Decision-Theoretic Sensitivity Analysis for Complex Computer Models, Technometrics 51 (2009) $121-129$. 